\title{
Propagation of gravitational waves in Chern-Simons axion Einstein gravity
}

\author{
Shin'ichi Nojiri, ${ }^{1,2, *}$ S. D. Odintsov, ${ }^{3,4,5, \dagger}$ V. K. Oikonomou $\odot,{ }^{6,7,8, \hbar}$ and Arkady A. Popov $\circledast^{9, \S}$ \\ ${ }^{1}$ Department of Physics, Nagoya University, Nagoya 464-8602, Japan \\ ${ }^{2}$ Kobayashi-Maskawa Institute for the Origin of Particles and the Universe, Nagoya University, \\ Nagoya 464-8602, Japan \\ ${ }^{3}$ ICREA, Passeig Luis Companys, 23, 08010 Barcelona, Spain \\ ${ }^{4}$ Institute of Space Sciences (IEEC-CSIC) C. Can Magrans s/n, 08193 Barcelona, Spain \\ ${ }^{5}$ Tomsk State Pedagogical University, 634061 Tomsk, Russia \\ ${ }^{6}$ Department of Physics, Aristotle University of Thessaloniki, Thessaloniki 54124, Greece \\ ${ }^{7}$ International Laboratory for Theoretical Cosmology, Tomsk State University of Control Systems \\ and Radioelectronics (TUSUR), 634050 Tomsk, Russia \\ ${ }^{8}$ Theoretical Astrophysics, IAAT, University of Tübingen, Tuebingen, Germany \\ ${ }^{9}$ N. I. Lobachevsky Institute of Mathematics and Mechanics, Kazan Federal University, \\ 420008, Kremlevskaya street 18, Kazan, Russia
}

(Received 12 August 2019; published 8 October 2019)

\begin{abstract}
In this paper we shall investigate the propagation of gravitational waves in a flat Friedman-RobertsonWalker background, in the context of a string motivated corrected Einstein gravity. Particularly, we shall consider a misalignment axion Einstein gravity in the presence of a string originating Chern-Simons coupling of the axion field to the Chern-Pontryagin density in four dimensions. We shall focus our study on the propagation of the gravitational waves, and we shall investigate whether there exists any difference in the propagation of the polarization states of the gravitational waves. As we demonstrate, the dispersion relations are different in the right-handed mode and the left-handed mode. Finally, we compare the propagation of the axion Chern-Simons Einstein theory with that of standard $F(R)$ gravity.
\end{abstract}

DOI: $10.1103 /$ PhysRevD.100.084009

\section{INTRODUCTION}

String theory is one solid theoretical framework that may describe, in a consistent way, the ultraviolet completion of classical gravity and of the Standard Model of particle physics. In some cases, several low-energy string theory effects may have their impact in the classical gravitational phenomena. One such example is offered by axionlike particles [1-11], and specifically from the misalignment axions [1]. For these axions, there exists a primordial $U(1)$ symmetry which is unbroken quite earlier from the inflationary era, but it is broken during the inflationary era. The cosmological implications of the axions have quite appealing features and, in view of the growing research stream that is related with axion experiments and observations ([12-22] see also [23]), axion physics has become quite timely. Actually, the axion may be one of the last realistic weakly interacting massive particles (WIMP) [24], since a longstanding number of experiments focusing on large WIMP masses ended up with no result. Thus, unless

\footnotetext{
*nojiri@gravity.phys.nagoya-u.ac.jp

†odintsov@ieec.uab.es

*v.k.oikonomou1979@gmail.com

§arkady_popov@mail.ru
}

supersymmetry is discovered in the Large Hadron Collider, the axion seems to be the last realistic particle dark matter candidate.

Very closely related to axion gravity, the so-called ChernSimons term [25-41], coupled with a function depending on the axion field, may be a realistic string theory originating correction, which may have a direct impact on low-energy gravitational phenomena and even in the inflationary era itself. The Chern-Simons term coupled to the axion has the form $U(\phi) \tilde{R} R$, and it is just the Chern-Pontryagin density. This string motivated term can have quite interesting effects on inflationary physics, as it was demonstrated first in Refs. [42,43]; see also Refs. [3-5] for some recent modified gravity applications. As it was shown in [42], the ChernSimons term can affect directly the tensor modes of the primordial perturbations, and this can have a measurable effect on the tensor-to-scalar ratio. This feature was also shown to occur in the context of the Chern-Simons $F(R)$ gravity [3]. Also in Ref. [43], it was demonstrated that the primordial gravitational waves have two polarizations that propagate in a different way. This phenomenon is similar and is related to chiral gravitational waves, which are also a very timely subject of current research [44-51].

Motivated by the above, in this work we shall investigate the propagation of gravitational waves, in the presence of 
the Chern-Simons axion term in the context of Einstein gravity. Our aim is to discover whether the dispersion relations are different for the two polarization modes, that is, the right-handed mode and the left-handed mode, which are given by the linear combinations of the + mode and $x$ mode, focusing on the present time era, since it is known that after horizon crossing, the amplitudes of the gravitational waves are conserved in the large scale limit [43]. Our findings are quite interesting, since we demonstrate that during a late-time nearly de Sitter era, if the axion coupling term is nearly constant, there exists a nontrivial mixing between the polarization states, and the two polarizations have different dispersion relations. In addition, just for comparison reasons, we discuss the propagation of gravitational waves in the context of $F(R)$ gravity, which is the most representative theory of modified gravity [52-58], and we compare the results to the Chern-Simons axion Einstein theory.
This paper is organized as follows: In Sec. II, we discuss several geometric features of the Einstein Chern-Simons gravity, which we shall extensively use in the next sections. In Sec. III, we present the essential features of the Einstein Chern-Simons theory, and we study in detail the propagation of gravitational waves in a flat Friedmann-RobertsonWalker (FRW) background. Finally, in Sec. IV, we discuss in detail the propagation of gravitational waves in $F(R)$ gravity, and we compare the results of $F(R)$ gravity with the results we found corresponding to the Einstein ChernSimons gravity.

\section{GEOMETRIC BACKGROUND AND FORMALISM}

Before getting to the core of our study, we shall briefly elaborate on the geometric features of the theory at hand. The curvature tensors and the connections are

$$
R=g^{\mu \nu} R_{\mu \nu}, \quad R_{\mu \nu}=R_{\mu \lambda \nu}^{\lambda}, \quad R_{\mu \rho \nu}^{\lambda}=-\Gamma_{\mu \rho, \nu}^{\lambda}+\Gamma_{\mu \nu, \rho}^{\lambda}-\Gamma_{\mu \rho}^{\eta} \Gamma_{\nu \eta}^{\lambda}+\Gamma_{\mu \nu}^{\eta} \Gamma_{\rho \eta}^{\lambda}, \quad \Gamma_{\mu \lambda}^{\eta}=\frac{1}{2} g^{\eta \nu}\left(g_{\mu \nu, \lambda}+g_{\lambda \nu, \mu}-g_{\mu \lambda, \nu}\right) .
$$

By varying the metric tensor $g_{\mu \nu}$ as follows:

$$
g_{\mu \nu} \rightarrow g_{\mu \nu}+\delta g_{\mu \nu}
$$

we obtain the variation of the following tensors, which we shall need and use extensively in the following sections:

$$
\begin{aligned}
\delta \Gamma_{\mu \nu}^{\kappa} & =\frac{1}{2} g^{\kappa \lambda}\left(\nabla_{\mu} \delta g_{\nu \lambda}+\nabla_{\nu} \delta g_{\mu \lambda}-\nabla_{\lambda} \delta g_{\mu \nu}\right), \\
\delta R_{\nu \lambda \sigma}^{\mu} & =\nabla_{\lambda} \delta \Gamma_{\sigma \nu}^{\mu}-\nabla_{\sigma} \delta \Gamma_{\lambda \nu}^{\mu}, \\
\delta R_{\mu \nu \lambda \sigma} & =\frac{1}{2}\left[\nabla_{\lambda} \nabla_{\nu} \delta g_{\sigma \mu}-\nabla_{\lambda} \nabla_{\mu} \delta g_{\sigma \nu}-\nabla_{\sigma} \nabla_{\nu} \delta g_{\lambda \mu}+\nabla_{\sigma} \nabla_{\mu} \delta g_{\lambda \nu}+\delta g_{\mu \rho} R_{\nu \lambda \sigma}^{\rho}-\delta g_{\nu \rho} R^{\rho}{ }_{\mu \lambda \sigma}\right], \\
\delta R_{\mu \nu} & =\frac{1}{2}\left[\nabla^{\rho}\left(\nabla_{\mu} \delta g_{\nu \rho}+\nabla_{\nu} \delta g_{\mu \rho}\right)-\nabla^{2} \delta g_{\mu \nu}-\nabla_{\mu} \nabla_{\nu}\left(g^{\rho \lambda} \delta g_{\rho \lambda}\right)\right] \\
& =\frac{1}{2}\left[\nabla_{\mu} \nabla^{\rho} \delta g_{\nu \rho}+\nabla_{\nu} \nabla^{\rho} \delta g_{\mu \rho}-\nabla^{2} \delta g_{\mu \nu}-\nabla_{\mu} \nabla_{\nu}\left(g^{\rho \lambda} \delta g_{\rho \lambda}\right)-2 R_{\nu}^{\lambda}{ }_{\mu} \delta g_{\lambda \rho}+R_{\mu}^{\rho} \delta g_{\rho \nu}+R_{\mu}^{\rho} \delta g_{\rho \nu}\right], \\
\delta R & =-\delta g_{\mu \nu} R^{\mu \nu}+\nabla^{\mu} \nabla^{\nu} \delta g_{\mu \nu}-\nabla^{2}\left(g^{\mu \nu} \delta g_{\mu \nu}\right), \\
{\left[\nabla_{\mu}, \nabla_{\nu}\right] \delta g_{\lambda \sigma} } & =-\delta g_{\rho \sigma} R^{\rho}{ }_{\lambda \mu \nu}-\delta g_{\lambda \rho} R^{\rho}{ }_{\sigma \mu \nu} .
\end{aligned}
$$

Consider now the antisymmetric tensor $A_{\mu \nu \rho \sigma}$, and with it we construct the following action:

$$
S_{A}=\int d x^{\mu} d x^{\nu} d x^{\rho} d x^{\sigma} A_{\mu \nu \rho \sigma}
$$

which is invariant under the general coordinate transformation. If we define the totally antisymmetric LeviCivita symbols $\epsilon_{\mu \nu \rho \sigma}$ and $\epsilon^{\mu \nu \rho \sigma}$ as follows:

$$
\epsilon_{0123}=-\epsilon^{0123}=1
$$

we have the following:

$$
\begin{aligned}
d x^{\mu} d x^{\nu} d x^{\rho} d x^{\sigma} A_{\mu \nu \rho \sigma} & =-\frac{1}{4 !} d x^{\mu} d x^{\nu} d x^{\rho} d x^{\sigma} \epsilon_{\mu \nu \rho \sigma^{\prime}} A_{\mu^{\prime} \nu^{\prime} \rho^{\prime} \sigma^{\prime}} \epsilon^{\mu^{\prime} \nu^{\prime} \rho^{\prime} \sigma^{\prime}} \\
& =d^{4} x \sqrt{-g} \frac{1}{\sqrt{-g}} A_{\mu^{\prime} \nu^{\prime} \rho^{\prime} \sigma^{\prime}} \epsilon^{\mu^{\prime} \nu^{\prime} \rho^{\prime} \sigma^{\prime}}
\end{aligned}
$$

Then, if we define $\tilde{\epsilon}^{\mu \nu \rho \sigma}$ by 


$$
\tilde{\epsilon}^{\mu \nu \rho \sigma} \equiv \frac{1}{\sqrt{-g}} \epsilon^{\mu \nu \rho \sigma}
$$

we can regard $\tilde{\epsilon}^{\mu \nu \rho \sigma}$ as a tensor. We can also define

$$
\epsilon_{\mu \nu \rho \sigma}=\eta_{\mu \mu^{\prime}} \eta_{\nu \nu^{\prime}} \eta_{\rho \rho^{\prime}} \eta_{\sigma \sigma^{\prime}} \epsilon^{\mu^{\prime} \nu^{\prime} \rho^{\prime} \sigma^{\prime}}
$$

and in addition,

$$
\tilde{\epsilon}_{\mu \nu \rho \sigma} \equiv g_{\mu \mu^{\prime}} g_{\nu \nu^{\prime}} g_{\rho \rho^{\prime}} g_{\sigma \sigma^{\prime}} \tilde{\epsilon}^{\mu^{\prime} \nu^{\prime} \rho^{\prime} \sigma^{\prime}}=\sqrt{-g} \epsilon_{\mu \nu \rho \sigma}
$$

We should note that

$$
\nabla_{\sigma} \tilde{\epsilon}^{\zeta \eta \rho \xi}=0
$$

due to the fact that

$$
\begin{aligned}
\tilde{\epsilon}_{\zeta \eta \rho \xi} \nabla_{\sigma} \tilde{\epsilon}^{\zeta \eta \rho \xi} & =\tilde{\epsilon}_{\zeta \eta \rho \xi}\left(\partial_{\sigma} \tilde{\epsilon}^{\zeta \eta \rho \xi}+\Gamma_{\sigma \tau}^{\zeta} \tilde{\epsilon}^{\tau \eta \rho \xi}+\Gamma_{\sigma \tau}^{\eta} \tilde{\epsilon}^{\zeta \tau \rho \xi}+\Gamma_{\sigma \tau}^{\rho} \tilde{\epsilon}^{\zeta \eta \tau \tau}+\Gamma_{\sigma \tau}^{\xi} \tilde{\epsilon}^{\zeta \eta \rho \tau}\right) \\
& =-\frac{4 !}{2} g^{\mu \nu} \partial_{\sigma} g_{\mu \nu}+3 !\left(\Gamma_{\sigma \zeta}^{\zeta}+\Gamma_{\sigma \eta}^{\eta}+\Gamma_{\sigma \rho}^{\rho}+\Gamma_{\sigma \xi}^{\xi}\right) \\
& =\frac{4 !}{2}\left(-g^{\mu \nu} \partial_{\sigma} g_{\mu \nu}+g^{\tau \mu}\left(\partial_{\sigma} g_{\mu \tau}+\partial_{\tau} g_{\sigma \mu}-\partial_{\mu} g_{\sigma \tau}\right)\right) \\
& =0 .
\end{aligned}
$$

We should note that there is only one nonvanishing component in $\tilde{\epsilon}^{\zeta \eta \rho \xi}$ and therefore if $\tilde{\epsilon}_{\zeta \eta \rho \xi} \nabla_{\sigma} \tilde{\epsilon}^{\zeta \eta \rho \xi}$ vanishes, the quantity $\nabla_{\sigma} \tilde{\epsilon}^{\zeta \eta \rho \xi}$ also vanishes. The above identities and quantities defined shall be extensively used in the following section.

\section{THE CHERN-SIMONS CORRECTED AXION MODEL}

We shall consider a Chern-Simons corrected misalignment axion in the context of Einstein gravity, so the action has the following form:

$$
S=\frac{1}{2 \kappa^{2}} \int d^{4} x \sqrt{-g}\left[R-\frac{\omega(\phi)}{2} \partial_{\mu} \phi \partial^{\mu} \phi-V(\phi)+U(\phi) \tilde{\epsilon}^{\mu \nu \rho \sigma} R_{\lambda \mu \nu}^{\tau} R_{\tau \rho \sigma}^{\lambda}\right] .
$$

In most cases, the misalignment axion is taken to be a canonical scalar field, but we assume that it has a generalized kinetic term, for generality of the argument. Because of the fact that

$$
\delta\left(\sqrt{-g} U(\phi) \tilde{\epsilon}^{\mu \nu \rho \sigma} R_{\lambda \mu \nu}^{\tau} R^{\lambda} \tau \rho \sigma\right)=2 \sqrt{-g} U(\phi)\left[\tilde{\epsilon}^{\zeta \eta \rho \mu} R_{\zeta \eta}^{\tau \nu}+\tilde{\epsilon}^{\zeta \eta \rho \nu} R^{\tau \mu}{ }_{\zeta \eta}\right] \nabla_{\rho} \nabla_{\tau} \delta g_{\mu \nu}
$$

by varying Eq. (2), the action (12) gives the following equations of motion:

$$
0=\frac{1}{2}\left\{R-\frac{\omega(\phi)}{2} \partial_{\mu} \phi \partial^{\mu} \phi-V(\phi)\right\} g_{\mu \nu}-R_{\mu \nu}+\frac{\omega(\phi)}{2} \partial_{\mu} \phi \partial_{\nu} \phi+2\left(g_{\mu \xi} g_{\nu \sigma}+g_{\mu \sigma} g_{\nu \xi}\right) \nabla_{\tau} \nabla_{\rho}\left(U(\phi) \tilde{\epsilon}^{\xi \eta \rho \xi} R_{\zeta \eta}^{\tau \sigma}\right) .
$$

Let us in brief consider the solutions in a flat FRW spacetime, the line element of which is

$$
d s^{2}=-d t^{2}+a(t)^{2} \sum_{i=1,2,3}\left(d x^{i}\right)^{2},
$$

and we shall assume that the scalar field $\phi$ depends solely on the cosmic time $t$. Then, in view of the following relations that hold true for the flat FRW background:

$$
\begin{aligned}
& \Gamma_{i j}^{t}=a^{2} H \delta_{i j}, \quad \Gamma_{j t}^{i}=\Gamma_{t j}^{i}=H \delta^{i}{ }_{j}, \quad \Gamma_{j k}^{i}=\tilde{\Gamma}_{j k}^{i}, \quad R_{i t j t}=-\left(\dot{H}+H^{2}\right) a^{2} \delta_{i j}, \quad R_{i j k l}=a^{4} H^{2}\left(\delta_{i k} \delta_{l j}-\delta_{i l} \delta_{k j}\right), \\
& R_{t t}=-3\left(\dot{H}+H^{2}\right), \quad R_{i j}=a^{2}\left(\dot{H}+3 H^{2}\right) \delta_{i j}, \quad R=6 \dot{H}+12 H^{2}, \quad \text { other components }=0,
\end{aligned}
$$

we obtain the following two equations:

$$
\begin{aligned}
& 0=-3 H^{2}+\frac{\omega(\phi)}{4} \dot{\phi}^{2}+\frac{V(\phi)}{2}, \\
& 0=2 \dot{H}+3 H^{2}+\frac{\omega(\phi)}{4} \dot{\phi}^{2}-\frac{V(\phi)}{2} .
\end{aligned}
$$

We should note that the term containing the scalar coupling function to the Chern-Simons term, namely, $U(\phi)$, does not contribute to the above equations in (17). This was also noted in Ref. [42] and actually the Chern-Simons does not affect the scalar perturbations at all, only the tensor perturbations. We may choose $\phi$ to be the cosmological time $t$, that is, $\phi=t$. Then the equations in (17) have the following forms: 


$$
\begin{aligned}
& 0=-3 H^{2}+\frac{\omega(\phi)}{4}+\frac{V(\phi)}{2}, \\
& 0=2 \dot{H}+3 H^{2}+\frac{\omega(\phi)}{4}-\frac{V(\phi)}{2},
\end{aligned}
$$

which can be solved with respect to $\omega(\phi)$ and $V(\phi)$ as follows:

$$
\omega(\phi)=-4 \dot{H}, \quad V(\phi)=2 \dot{H}+6 H^{2} .
$$

Then if we choose

$\omega(\phi)=-4 f^{\prime}(\phi), \quad V(\phi)=2 f^{\prime}(\phi)+6 f(\phi)^{2}$,

a solution of the equations in (17) is given by $H=f(t)$ and $\phi=t$. After these preliminary general features, which indicated that the Chern-Simons term does not affect at all the equations of motion of the gravitational theory, we proceed to the study of the gravity waves. As we shall demonstrate in the next subsection, the gravitational waves are affected significantly by the presence of the nontrivial Chern-Simons term.

\section{A. Gravitational waves and their polarizations}

In this section we shall study the propagation of gravitational waves in the Chern-Simons axion Einstein gravity. In order to study the propagation of the gravitational wave, we consider the perturbation of Eq. (14), from the background whose metric is $g_{\mu \nu}^{(0)}$,

$$
g_{\mu \nu}=g_{\mu \nu}^{(0)}+h_{\mu \nu} .
$$

Then the corresponding Einstein equations are

$$
\begin{aligned}
G_{\mu \nu}= & -\frac{1}{2}\left(\nabla_{\mu}^{(0)} \nabla^{(0) \rho} h_{\nu \rho}+\nabla_{\nu}^{(0)} \nabla^{(0) \rho} h_{\mu \rho}-\square^{(0)} h_{\mu \nu}-\nabla_{\mu}^{(0)} \nabla_{\nu}^{(0)}\left(g^{(0) \rho \lambda} h_{\rho \lambda}\right)-2 R_{\nu}^{(0) \lambda}{ }_{\mu} h_{\lambda \rho}+R_{\mu}^{(0) \rho} h_{\rho \nu}+R_{\nu}^{(0) \rho} h_{\rho \mu}\right) \\
& +\frac{1}{2}\left(R^{(0)}-\frac{\omega\left(\phi^{(0)}\right)}{2} g^{(0) \rho \sigma} \partial_{\rho} \phi^{(0)} \partial_{\sigma} \phi^{(0)}-V\left(\phi^{(0)}\right)\right) h_{\mu \nu}+\frac{1}{2} g_{\mu \nu}^{(0)}\left(-h_{\rho \sigma}\left(R^{(0) \rho \sigma}-\frac{\omega\left(\phi^{(0)}\right)}{2} \partial^{\rho} \phi^{(0)} \partial^{\sigma} \phi^{(0)}\right)\right. \\
& \left.+\nabla^{(0) \rho} \nabla^{(0) \sigma} h_{\rho \sigma}-\square^{(0)}\left(g^{(0) \rho \sigma} h_{\rho \sigma}\right)\right)+2\left(h_{\mu \xi} g_{\nu \sigma}^{(0)}+h_{\mu \sigma} g_{\nu \xi}^{(0)}+g_{\mu \xi}^{(0)} h_{\nu \sigma}+g_{\mu \sigma}^{(0)} h_{\nu \xi}\right) \tilde{\epsilon}^{(0) \zeta \eta \rho \xi} \nabla_{\tau}^{(0)} \nabla_{\rho}^{(0)}\left(U\left(\phi^{(0)}\right) R^{(0) \tau \sigma}{ }_{\zeta \eta}\right) \\
& +2\left(g_{\mu \xi}^{(0)} g_{\nu \sigma}^{(0)}+g_{\mu \sigma}^{(0)} g_{\nu \xi}^{(0)}\right)\left\{-\frac{1}{2} g^{(0) \alpha \beta} h_{\alpha \beta} \tilde{\epsilon}^{(0) \zeta \eta \rho \xi} \nabla_{\tau}^{(0)} \nabla_{\rho}^{(0)}\left(U\left(\phi^{(0)}\right) R^{(0) \tau \sigma}{ }_{\zeta \eta}\right)+\tilde{\epsilon}^{(0) \zeta \eta \rho \xi} h_{\alpha \beta} \nabla^{(0) \alpha} \nabla_{\rho}^{(0)}\left(U\left(\phi^{(0)}\right) R^{(0) \sigma \beta}{ }_{\zeta \eta}\right)\right. \\
& -\frac{1}{2} \tilde{\epsilon}^{(0) \zeta \eta \rho \xi} g^{(0) \tau \alpha} \nabla_{\tau}^{(0)} \nabla_{\rho}^{(0)}\left(2 U\left(\phi^{(0)}\right) g^{(0) \sigma \beta}\left(\nabla_{\zeta}^{(0)}\left(\nabla_{\eta}^{(0)} h_{\alpha \beta}+\nabla_{\alpha}^{(0)} h_{\eta \beta}-\nabla_{\beta}^{(0)} h_{\eta \alpha}\right)\right)\right) \\
& -\frac{1}{2} \tilde{\epsilon}^{(0) \zeta \eta \rho \xi} g^{(0) \tau \alpha} \nabla_{\tau}^{(0)}\left(U ( \phi ^ { ( 0 ) } ) \left(g^{(0) \sigma \beta}\left(\nabla_{\rho}^{(0)} h_{\beta \gamma}+\nabla_{\gamma}^{(0)} h_{\rho \beta}-\nabla_{\beta}^{(0)} h_{\rho \gamma}\right) R^{(0) \gamma}{ }_{\alpha \zeta \eta}\right.\right. \\
& -g^{(0) \gamma \beta}\left(\nabla_{\rho}^{(0)} h_{\beta \alpha}+\nabla_{\alpha}^{(0)} h_{\rho \beta}-\nabla_{\beta}^{(0)} h_{\rho \alpha}\right) R^{(0) \sigma}{ }_{\gamma \zeta \eta}-g^{(0) \gamma \beta}\left(\nabla_{\rho}^{(0)} h_{\beta \zeta}+\nabla_{\zeta}^{(0)} h_{\rho \beta}-\nabla_{\beta}^{(0)} h_{\rho \zeta}\right) R^{(0) \sigma}{ }_{\alpha \gamma \eta} \\
& \left.\left.-g^{(0) \gamma \beta}\left(\nabla_{\rho}^{(0)} h_{\beta \eta}+\nabla_{\eta}^{(0)} h_{\rho \beta}-\nabla_{\beta}^{(0)} h g_{\rho \eta}\right) R^{(0) \sigma}{ }_{\alpha \zeta \gamma}\right)\right) \\
& -\frac{1}{2} \tilde{\epsilon}^{(0) \zeta \eta \rho \xi} g^{(0) \tau \alpha}\left(-g^{(0) \beta \gamma}\left(\nabla_{\tau}^{(0)} h_{\gamma \rho}+\nabla_{\rho}^{(0)} h_{\tau \gamma}-\nabla_{\gamma}^{(0)} h_{\tau \rho}\right) \nabla_{\beta}^{(0)}\left(U\left(\phi^{(0)}\right) R^{(0) \sigma}{ }_{\alpha \zeta \eta}\right)\right. \\
& +g^{(0) \sigma \gamma}\left(\nabla_{\tau}^{(0)} h_{\gamma \beta}+\nabla_{\beta}^{(0)} h_{\tau \gamma}-\nabla_{\gamma}^{(0)} h_{\tau \beta}\right) \nabla_{\rho}^{(0)}\left(U\left(\phi^{(0)}\right) R^{(0) \beta}{ }_{\alpha \zeta \eta}\right) \\
& -g^{(0) \beta \gamma}\left(\nabla_{\tau}^{(0)} h_{\gamma \alpha}+\nabla_{\alpha}^{(0)} h_{\tau \gamma}-\nabla_{\gamma}^{(0)} h_{\tau \alpha}\right) \nabla_{\rho}^{(0)}\left(U\left(\phi^{(0)}\right) R_{\beta \zeta \eta}^{(0) \sigma}\right) \\
& -g^{(0) \beta \gamma}\left(\nabla_{\tau}^{(0)} h_{\gamma \zeta}+\nabla_{\zeta}^{(0)} h_{\tau \gamma}-\nabla_{\gamma}^{(0)} h_{\tau \zeta}\right) \nabla_{\rho}^{(0)}\left(U\left(\phi^{(0)}\right) R^{(0) \sigma}{ }_{\alpha \beta \eta}\right) \\
& \left.\left.-g^{(0) \beta \gamma}\left(\nabla_{\tau}^{(0)} h_{\gamma \eta}+\nabla_{\eta}^{(0)} h_{\tau \gamma}-\nabla_{\gamma}^{(0)} h_{\tau \eta}\right) \nabla_{\rho}^{(0)}\left(U\left(\phi^{(0)}\right) R^{(0) \sigma}{ }_{\alpha \zeta \beta}\right)\right)\right\}=0 .
\end{aligned}
$$

In the Appendix, we present the explicit form of the $(t, t)$, $(i, j)$, and $(t, i)$ components of the Einstein tensor of Eq. (22) in the FRW background (15). We now choose the following gauge condition:

$$
0=\nabla^{\mu} h_{\mu \nu} .
$$

In the FRW background (15), the gauge condition (23) has the following forms:

$$
\begin{aligned}
0= & -3 H h_{t t}-\frac{\partial h_{t t}}{\partial t}-\frac{H}{a^{2}}\left(h_{x x}+h_{y y}+h_{z z}\right) \\
& +\frac{1}{a^{2}}\left(\frac{\partial h_{t x}}{\partial x}+\frac{\partial h_{t y}}{\partial y}+\frac{\partial h_{t z}}{\partial z}\right),
\end{aligned}
$$


$0=-3 H h_{t x}-\frac{\partial h_{t x}}{\partial t}+\frac{1}{a^{2}}\left(\frac{\partial h_{x x}}{\partial x}+\frac{\partial h_{x y}}{\partial y}+\frac{\partial h_{x z}}{\partial z}\right)$,

$0=-3 H h_{t y}-\frac{\partial h_{t y}}{\partial t}+\frac{1}{a^{2}}\left(\frac{\partial h_{y y}}{\partial y}+\frac{\partial h_{x y}}{\partial x}+\frac{\partial h_{y z}}{\partial z}\right)$,

$0=-3 H h_{t z}-\frac{\partial h_{t z}}{\partial t}+\frac{1}{a^{2}}\left(\frac{\partial h_{z z}}{\partial z}+\frac{\partial h_{x z}}{\partial x}+\frac{\partial h_{y z}}{\partial y}\right)$.

As we are interested in the gravitational wave, which corresponds to the massless spin-two mode, we assume that

$$
h_{t \mu}=h_{\mu t}=0, \quad \sum_{i=1,2,3} h_{i i}=0 .
$$

Then Eqs. (24), (25), (26), and (27) yield

$$
0=\sum_{j} \partial_{j} h_{j i}
$$

In effect, Eq. (A1) in the Appendix indicates that $G_{t t}=G_{t x}=G_{t y}=G_{t z}=0$, that is, $(t, t),(t, x),(t, y)$, and $(t, z)$ components of (22) are satisfied. In addition, the other Einstein tensor components are

$$
\begin{aligned}
& G_{x x}=\dot{H} h_{x x}+H^{2} h_{x x}-\frac{1}{2} \frac{\partial^{2} h_{x x}}{\partial t^{2}}+H \frac{1}{2} \frac{\partial h_{x x}}{\partial t}+\frac{1}{a^{2}}\left(\frac{\partial^{2} h_{y z}}{\partial z \partial y}-\frac{1}{2} \frac{\partial^{2} h_{y y}}{\partial z^{2}}-\frac{1}{2} \frac{\partial^{2} h_{z z}}{\partial y^{2}}\right) \\
& +\frac{8 \dot{H} \dot{U}}{a}\left(\frac{\partial h_{x z}}{\partial y}-\frac{\partial h_{x y}}{\partial z}\right)+H\left[\frac{8 \ddot{U}}{a}\left(\frac{\partial h_{x z}}{\partial y}-\frac{\partial h_{x y}}{\partial z}\right)+\frac{8 \dot{U}}{a}\left(\frac{\partial^{2} h_{x z}}{\partial y \partial t}-\frac{\partial^{2} h_{x y}}{\partial z \partial t}\right)\right] \\
& +\frac{4 \dot{U}}{a^{3}}\left(\frac{\partial^{3} h_{x z}}{\partial y^{3}}-\frac{\partial^{3} h_{x y}}{\partial z^{3}}-\frac{\partial^{3} h_{x y}}{\partial z \partial y^{2}}+\frac{\partial^{3} h_{x z}}{\partial y \partial z^{2}}-\frac{\partial^{3} h_{y z}}{\partial x \partial y^{2}}+\frac{\partial^{3} h_{y z}}{\partial x \partial z^{2}}+\frac{\partial^{3}\left(h_{y y}-h_{z z}\right)}{\partial x \partial y \partial z}\right) \\
& +\frac{4 \ddot{U}}{a}\left(\frac{\partial^{2} h_{x y}}{\partial z \partial t}-\frac{\partial^{2} h_{x z}}{\partial y \partial t}\right)+\frac{4 \dot{U}}{a}\left(-\frac{\partial^{3} h_{x z}}{\partial y \partial t^{2}}+\frac{\partial^{3} h_{x y}}{\partial z \partial t^{2}}\right), \\
& G_{y y}=\dot{H} h_{y y}+H^{2} h_{y y}-\frac{1}{2} \frac{\partial^{2} h_{y y}}{\partial t^{2}}+H \frac{1}{2} \frac{\partial h_{y y}}{\partial t}+\frac{1}{a^{2}}\left(\frac{\partial^{2} h_{z x}}{\partial x \partial z}-\frac{1}{2} \frac{\partial^{2} h_{z z}}{\partial x^{2}}-\frac{1}{2} \frac{\partial^{2} h_{x x}}{\partial z^{2}}\right) \\
& +\frac{8 \dot{H} \dot{U}}{a}\left(\frac{\partial h_{y x}}{\partial z}-\frac{\partial h_{y z}}{\partial x}\right)+H\left[\frac{8 \ddot{U}}{a}\left(\frac{\partial h_{y x}}{\partial z}-\frac{\partial h_{y z}}{\partial x}\right)+\frac{8 \dot{U}}{a}\left(\frac{\partial^{2} h_{y x}}{\partial z \partial t}-\frac{\partial^{2} h_{y z}}{\partial x \partial t}\right)\right] \\
& +\frac{4 \dot{U}}{a^{3}}\left(\frac{\partial^{3} h_{y x}}{\partial z^{3}}-\frac{\partial^{3} h_{y z}}{\partial x^{3}}-\frac{\partial^{3} h_{y z}}{\partial x \partial z^{2}}+\frac{\partial^{3} h_{y x}}{\partial z \partial x^{2}}-\frac{\partial^{3} h_{z x}}{\partial y \partial z^{2}}+\frac{\partial^{3} h_{z x}}{\partial y \partial x^{2}}+\frac{\partial^{3}\left(h_{z z}-h_{x x}\right)}{\partial y \partial z \partial x}\right) \\
& +\frac{4 \ddot{U}}{a}\left(\frac{\partial^{2} h_{y z}}{\partial x \partial t}-\frac{\partial^{2} h_{y x}}{\partial z \partial t}\right)+\frac{4 \dot{U}}{a}\left(-\frac{\partial^{3} h_{y x}}{\partial z \partial t^{2}}+\frac{\partial^{3} h_{y z}}{\partial x \partial t^{2}}\right), \\
& G_{z z}=\dot{H} h_{z z}+H^{2} h_{z z}-\frac{1}{2} \frac{\partial^{2} h_{z z}}{\partial t^{2}}+H \frac{1}{2} \frac{\partial h_{z z}}{\partial t}+\frac{1}{a^{2}}\left(\frac{\partial^{2} h_{x y}}{\partial y \partial x}-\frac{1}{2} \frac{\partial^{2} h_{x x}}{\partial y^{2}}-\frac{1}{2} \frac{\partial^{2} h_{y y}}{\partial x^{2}}\right) \\
& +\frac{8 \dot{H} \dot{U}}{a}\left(\frac{\partial h_{z y}}{\partial x}-\frac{\partial h_{z x}}{\partial y}\right)+H\left[\frac{8 \ddot{U}}{a}\left(\frac{\partial h_{z y}}{\partial x}-\frac{\partial h_{z x}}{\partial y}\right)+\frac{8 \dot{U}}{a}\left(\frac{\partial^{2} h_{z y}}{\partial x \partial t}-\frac{\partial^{2} h_{z x}}{\partial y \partial t}\right)\right] \\
& +\frac{4 \dot{U}}{a^{3}}\left(\frac{\partial^{3} h_{z y}}{\partial x^{3}}-\frac{\partial^{3} h_{z x}}{\partial y^{3}}-\frac{\partial^{3} h_{z x}}{\partial y \partial x^{2}}+\frac{\partial^{3} h_{z y}}{\partial x \partial y^{2}}-\frac{\partial^{3} h_{x y}}{\partial z \partial x^{2}}+\frac{\partial^{3} h_{x y}}{\partial z \partial y^{2}}+\frac{\partial^{3}\left(h_{x x}-h_{y y}\right)}{\partial z \partial x \partial y}\right) \\
& +\frac{4 \ddot{U}}{a}\left(\frac{\partial^{2} h_{z x}}{\partial y \partial t}-\frac{\partial^{2} h_{z y}}{\partial x \partial t}\right)+\frac{4 \dot{U}}{a}\left(-\frac{\partial^{3} h_{z y}}{\partial x \partial t^{2}}+\frac{\partial^{3} h_{z x}}{\partial y \partial t^{2}}\right), \\
& G_{x y}=\dot{H}\left[h_{x y}+\frac{4 \dot{U}}{a}\left(\frac{\partial\left(h_{x x}-h_{y y}\right)}{\partial z}+\frac{\partial h_{y z}}{\partial y}-\frac{\partial h_{x z}}{\partial x}\right)\right]+H^{2} h_{x y}+H\left[\frac{1}{2} \frac{\partial h_{x y}}{\partial t}+\frac{4 \ddot{U}}{a}\left(\frac{\partial\left(h_{x x}-h_{y y}\right)}{\partial z}+\frac{\partial h_{y z}}{\partial y}-\frac{\partial h_{x z}}{\partial x}\right)\right. \\
& \left.+\frac{4 \dot{U}}{a}\left(\frac{\partial^{2}\left(h_{x x}-h_{y y}\right)}{\partial z \partial t}+\frac{\partial^{2} h_{y z}}{\partial y \partial t}-\frac{\partial^{2} h_{x z}}{\partial x \partial t}\right)\right]-\frac{1}{2} \frac{\partial^{2} h_{x y}}{\partial t^{2}}+\frac{2 \ddot{U}}{a}\left(\frac{\partial^{2}\left(h_{y y}-h_{x x}\right)}{\partial z \partial t}+\frac{\partial^{2} h_{x z}}{\partial x \partial t}-\frac{\partial^{2} h_{y z}}{\partial y \partial t}\right) \\
& +\frac{2 \dot{U}}{a}\left(\frac{\partial^{3}\left(h_{y y}-h_{x x}\right)}{\partial z \partial t^{2}}+\frac{\partial^{3} h_{x z}}{\partial x \partial t^{2}}-\frac{\partial^{3} h_{y z}}{\partial y \partial t^{2}}\right)+\frac{1}{2 a^{2}}\left(\frac{\partial^{2} h_{x y}}{\partial z^{2}}+\frac{\partial^{2} h_{z z}}{\partial y \partial x}-\frac{\partial^{2} h_{x z}}{\partial z \partial y}-\frac{\partial^{2} h_{y z}}{\partial z \partial x}\right) \\
& +\frac{2 \dot{U}}{a^{3}}\left(\frac{\partial^{3}\left(h_{x x}-h_{y y}\right)}{\partial z^{3}}+\frac{\partial^{3}\left(h_{z z}-h_{y y}\right)}{\partial z \partial x^{2}}+\frac{\partial^{3}\left(h_{x x}-h_{z z}\right)}{\partial z \partial y^{2}}+2 \frac{\partial^{3} h_{y z}}{\partial y \partial x^{2}}-2 \frac{\partial^{3} h_{x z}}{\partial x \partial y^{2}}+2 \frac{\partial^{3} h_{y z}}{\partial y \partial z^{2}}-2 \frac{\partial^{3} h_{x z}}{\partial x \partial z^{2}}\right),
\end{aligned}
$$




$$
\begin{aligned}
G_{x z}= & \dot{H}\left[h_{x z}-\frac{4 \dot{U}}{a}\left(\frac{\partial\left(h_{x x}-h_{z z}\right)}{\partial y}+\frac{\partial h_{y z}}{\partial z}-\frac{\partial h_{x y}}{\partial x}\right)\right]+H^{2} h_{x z} \\
& +H\left[\frac{1}{2} \frac{\partial h_{x z}}{\partial t}-\frac{4 \ddot{U}}{a}\left(\frac{\partial\left(h_{x x}-h_{z z}\right)}{\partial y}+\frac{\partial h_{y z}}{\partial z}-\frac{\partial h_{x y}}{\partial x}\right)-\frac{4 \dot{U}}{a}\left(\frac{\partial^{2}\left(h_{x x}-h_{z z}\right)}{\partial y \partial t}+\frac{\partial^{2} h_{y z}}{\partial z \partial t}-\frac{\partial^{2} h_{x y}}{\partial x \partial t}\right)\right] \\
& -\frac{1}{2} \frac{\partial^{2} h_{x z}}{\partial t^{2}}-\frac{2 \ddot{U}}{a}\left(\frac{\partial^{2}\left(h_{z z}-h_{x x}\right)}{\partial y \partial t}+\frac{\partial^{2} h_{x y}}{\partial x \partial t}-\frac{\partial^{2} h_{y z}}{\partial z \partial t}\right)-\frac{2 \dot{U}}{a}\left(\frac{\partial^{3}\left(h_{z z}-h_{x x}\right)}{\partial y \partial t^{2}}+\frac{\partial^{3} h_{x y}}{\partial x \partial t^{2}}-\frac{\partial^{3} h_{y z}}{\partial z \partial t^{2}}\right) \\
& +\frac{1}{2 a^{2}}\left(\frac{\partial^{2} h_{x z}}{\partial y^{2}}+\frac{\partial^{2} h_{y y}}{\partial z \partial x}-\frac{\partial^{2} h_{x y}}{\partial z \partial y}-\frac{\partial^{2} h_{y z}}{\partial y \partial x}\right)-\frac{2 \dot{U}}{a^{3}}\left(\frac{\partial^{3}\left(h_{x x}-h_{z z}\right)}{\partial y^{3}}+\frac{\partial^{3}\left(h_{y y}-h_{z z}\right)}{\partial y \partial x^{2}}+\frac{\partial^{3}\left(h_{x x}-h_{y y}\right)}{\partial y \partial z^{2}}\right. \\
& \left.+2 \frac{\partial^{3} h_{y z}}{\partial z \partial x^{2}}-2 \frac{\partial^{3} h_{x y}}{\partial x \partial z^{2}}+2 \frac{\partial^{3} h_{y z}}{\partial z \partial y^{2}}-2 \frac{\partial^{3} h_{x y}}{\partial x \partial y^{2}}\right), \\
G_{y z}+\dot{H}\left[h_{y z}+\right. & \left.\frac{4 \dot{U}}{a}\left(\frac{\partial\left(h_{y y}-h_{z z}\right)}{\partial x}+\frac{\partial h_{x z}}{\partial z}-\frac{\partial h_{x y}}{\partial y}\right)\right]+H^{2} h_{y z}+H\left[\frac{1}{2} \frac{\partial h_{y z}}{\partial t}+\frac{4 \ddot{U}}{a}\left(\frac{\partial\left(h_{y y}-h_{z z}\right)}{\partial x}+\frac{\partial h_{x z}}{\partial z}-\frac{\partial h_{x y}}{\partial y}\right)\right. \\
+ & \left.\frac{4 \dot{U}}{a}\left(\frac{\partial^{2}\left(h_{y y}-h_{z z}\right)}{\partial x \partial t}+\frac{\partial^{2} h_{x z}}{\partial z \partial t}-\frac{\partial^{2} h_{x y}}{\partial y \partial t}\right)\right]-\frac{1}{2} \frac{\partial^{2} h_{y z}}{\partial t^{2}}+\frac{2 \ddot{U}}{a}\left(\frac{\partial^{2}\left(h_{z z}-h_{y y}\right)}{\partial x \partial t}+\frac{\partial^{2} h_{x y}}{\partial y \partial t}-\frac{\partial^{2} h_{x z}}{\partial z \partial t}\right) \\
+ & \frac{2 \dot{U}}{a}\left(\frac{\partial^{3}\left(h_{z z}-h_{y y}\right)}{\partial x \partial t^{2}}+\frac{\partial^{3} h_{x y}}{\partial y \partial t^{2}}-\frac{\partial^{3} h_{x z}}{\partial z \partial t^{2}}\right)+\frac{1}{2 a^{2}}\left(\frac{\partial^{2} h_{y z}}{\partial x^{2}}+\frac{\partial^{2} h_{x x}}{\partial z \partial y}-\frac{\partial^{2} h_{x y}}{\partial z \partial x}-\frac{\partial^{2} h_{x z}}{\partial y \partial x}\right) \\
+ & \frac{2 \dot{U}}{a^{3}}\left(\frac{\partial^{3}\left(h_{y y}-h_{z z}\right)}{\partial x^{3}}+\frac{\partial^{3}\left(h_{x x}-h_{z z}\right)}{\partial x \partial y^{2}}+\frac{\partial^{3}\left(h_{y y}-h_{x x}\right)}{\partial x \partial z^{2}}+2 \frac{\partial^{3} h_{x z}}{\partial z \partial y^{2}}-2 \frac{\partial^{3} h_{x y}}{\partial y \partial z^{2}}+2 \frac{\partial^{3} h_{x z}}{\partial z \partial x^{2}}-2 \frac{\partial^{3} h_{x y}}{\partial y \partial x^{2}}\right) .
\end{aligned}
$$

In order to reveal the propagation properties at present time, let us assume that the variations of the Hubble rate $H$ and of the axion Chern-Simons coupling function $\dot{U}$ during the propagation of the gravitational wave are negligible and they are constant, that is,

$$
H^{2}=H_{0}^{2}, \quad \dot{H}=H_{1}, \quad \dot{U}=U_{0}, \quad \ddot{U}=0,
$$

with constants $H_{0}, H_{1}$, and $U_{0}$. We also assume that the scale factor $a$ is slowly varying at present time, so we shall assume that it is approximately equal to unity, $a \simeq 1$. We also consider the gravitational wave propagating along the $z$ direction, that is, $h_{i j} \propto \mathrm{e}^{-i(\omega t-k z)}$, with a constant angular frequency $\omega$ and a constant wave number $k$. Then the conditions in (28) and (29) indicate that

$$
\begin{aligned}
& h_{i z}=0, \quad h_{x x}=-h_{y y}=h_{+} \mathrm{e}^{-i(\omega t-k z)}, \\
& h_{x y}=h_{y x}=h_{\times} \mathrm{e}^{-i(\omega t-k z)},
\end{aligned}
$$

with complex constants $h_{+}$and $h_{\times}$, which express the polarizations of the gravitational wave. Only the real parts in (37) are physically meaningful. Then, the nonzero components of the Einstein tensor are

$$
\begin{gathered}
G_{x x}=-G_{y y} \\
=\left(H_{1}+H_{0}^{2}-\frac{i \omega H_{0}}{2}+\frac{\omega^{2}}{2}-\frac{k^{2}}{2}\right) h_{+} \\
\quad-4 i k\left(2 H_{1}-2 i \omega H_{0}-k^{2}+\omega^{2}\right) U_{0} h_{\times}, \\
G_{x y}=\left(H_{1}+H_{0}^{2}-\frac{i \omega H_{0}}{2}+\frac{\omega^{2}}{2}-\frac{k^{2}}{2}\right) h_{\times} \\
+4 i k\left(2 H_{1}-2 i \omega H_{0}-k^{2}+\omega^{2}\right) U_{0} h_{+}, \\
G_{z z}=G_{x z}=G_{y z}=0 .
\end{gathered}
$$

An interesting situation arises when $U_{0} \neq 0$ and $H \neq 0$ $\left(H_{0} \neq 0\right.$ or $\left.H_{1} \neq 0\right)$, in which case there is always a mixing of the + mode corresponding to $h_{+}$and the $\times$mode to $h_{\times}$. This scenario is particularly interesting since there is a nontrivial polarization mode for the gravitational wave, and also the dispersion relation for the two polarization modes corresponding to the right-handed mode $h_{\times}=i h_{+}$and the left-handed mode $h_{\times}=-i h_{+}$are different from each other. We should note, however, even if $U_{0} \neq 0$, in a flat background where $H=0 \quad\left(H_{0}=H_{1}=0\right)$, as long as the dispersion relation $\omega^{2}=k^{2}$ is satisfied, the + mode and $\times$ mode become independent from each other.

We now explain the above mentioned things in more detail. From the condition that the equations $G_{x x}=G_{y y}=$ $G_{x y}=0$ have nontrivial solutions, we find the dispersion relation, 


$$
\begin{aligned}
& \left(H_{1}+H_{0}^{2}-\frac{i \omega H_{0}}{2}+\frac{\omega^{2}}{2}-\frac{k^{2}}{2}\right)^{2} \\
& =16 k^{2}\left(2 H_{1}-2 i \omega H_{0}+\omega^{2}-k^{2}\right)^{2} U_{0}^{2},
\end{aligned}
$$

that is,

$$
\begin{aligned}
H_{1} & +H_{0}^{2}-\frac{i \omega H_{0}}{2}+\frac{\omega^{2}}{2}-\frac{k^{2}}{2} \\
& = \pm 4 k\left(2 H_{1}-2 i \omega H_{0}+\omega^{2}-k^{2}\right) U_{0}
\end{aligned}
$$

which indicates that $h_{\times}= \pm i h_{+}$and if $k>0,+\operatorname{sign} h_{\times}=$ $i h_{+}$corresponds to the right-handed polarization and - sign $h_{\times}=i h_{+}$to the left-handed polarization (see [59], for example). Therefore the right-handed polarization mode has a dispersion relation different from that of the lefthanded polarization. The dispersion relation (42) can be rewritten in the following form:

$$
\begin{aligned}
0= & D_{L}(\omega, k) \equiv \omega^{2}-k^{2} \\
& +\frac{2 H_{1}+2 H_{0}^{2}-i \omega H_{0} \mp 16 k U_{0}\left(H_{1}-2 i \omega H_{0}\right)}{1 \mp 8 k U_{0}} .
\end{aligned}
$$

If we assume $\left|k U_{0}\right| \ll 1$, Eq. (45) has the following form:

$$
\begin{aligned}
0= & D_{L}(\omega, k) \\
\sim & \omega^{2}-k^{2}+2 H_{1}+2 H_{0}^{2}-i \omega H_{0} \pm 16 k U_{0} H_{0}^{2} \\
& \pm 24 i \omega k U_{0} H_{0} .
\end{aligned}
$$

Then, although the term $-i \omega H_{0}$ gives the dissipation coming from the expansion of the Universe, the last term $\pm 24 i \omega k U_{0} H_{0}$ works against for the dissipation in the righthanded mode (now we assume $k>0$ ) but works to increase the dissipation for the left-handed mode. When we consider the high frequency mode $\left|k U_{0}\right| \gg 1$ and $\omega H_{0} \gg H_{1}$, Eq. (45) has the following form:

$$
0=D_{L}(\omega, k) \sim \omega^{2}-k^{2}-4 i \omega H_{0} .
$$

We may compare the above expression with the expression in the case of $U_{0}=0$ and for the high frequency mode,

$$
0=D_{L}(\omega, k) \sim \omega^{2}-k^{2}-i \omega H_{0} .
$$

Although $U_{0}$ does not appear in the expression of Eq. (45), the dissipation is four times stronger than that in the case of $U_{0}=0$ in (46).

In general relativity, the gravitational wave has two modes corresponding to the helicity, that is, the righthanded mode and the left-handed mode. The two modes have the identical dispersion relation, which enables us to consider the + mode and $\times$ mode, instead of the right- and left-handed modes. In the model which we are now considering, we cannot consider the + mode and the $\times$ mode as independent modes because the right- and left-handed modes satisfy different dispersion relations, respectively. This is because the model breaks parity and therefore the model is chiral, which is an important result of this work. This polarization asymmetry of the two propagating modes of the gravitational wave could be detected in the future in the LIGO or the forthcoming LISA Collaborations. In addition, the polarization of the gravitational wave in the early Universe also affects the polarization of cosmic microwave background, and specifically the E mode and B modes; see, e.g., [60].

\section{GRAVITATIONAL WAVES IN $\boldsymbol{F}(R)$ GRAVITY}

For comparison reasons, in this section we shall study the gravitational waves in $F(R)$ gravity, with the action being

$$
S=\int d^{4} x \sqrt{-g}\left[\frac{F(R)}{2 \kappa^{2}}+\mathcal{L}_{\text {matter }}\left(g_{\mu \nu}, \Phi_{i}\right)\right]
$$

where $\mathcal{L}_{\text {matter }}$ is the Lagrangian density of the matter fluids present, and the $\Phi_{i}$ 's express the various different matter fields. We can rewrite the action (47) by introducing the auxiliary scalar fields $A$ and $B$ in the following way:

$$
\begin{aligned}
S_{A B}= & \int d^{4} x \sqrt{-g}\left[\frac{1}{2 \kappa^{2}}\{B(R-A)+F(A)\}\right. \\
& \left.+\mathcal{L}_{\text {matter }}\left(g_{\mu \nu}, \Phi_{i}\right)\right] .
\end{aligned}
$$

Upon variation of the action with respect to the auxiliary scalar $A$, we obtain

$$
B=F^{\prime}(A)
$$

By redefining the scalar field $B$ by introducing a new scalar field $\sigma$ with $B=\mathrm{e}^{\sigma}$, we assume that Eq. (49) can be solved with respect to $A$ as $A=A(\sigma)$. In effect, the action (48) can be rewritten as follows:

$$
\begin{aligned}
S_{\sigma \phi}= & \int d^{4} x \sqrt{-g}\left[\frac{1}{2 \kappa^{2}}\left\{\mathrm{e}^{\sigma}(R-A(\sigma))+F(A(\sigma))\right\}\right. \\
& \left.+\mathcal{L}_{\text {matter }}\left(g_{\mu \nu}, \Phi_{i}\right)\right] .
\end{aligned}
$$

By performing a scale transformation of the metric tensor,

$$
g_{\mu \nu}=\mathrm{e}^{-\sigma} \tilde{g}_{\mu \nu}
$$

the action (50) can be transformed in the Einstein frame, and it is equal to 


$$
\begin{aligned}
S_{\mathrm{E}}= & \int d^{4} x \sqrt{-\tilde{g}}\left[\frac{1}{2 \kappa^{2}}\left\{\tilde{R}-\frac{3}{2} \partial_{\mu} \sigma \partial^{\mu} \sigma\right\}\right. \\
& \left.-U(\sigma)+\mathrm{e}^{-2 \sigma} \mathcal{L}_{\text {matter }}\left(\mathrm{e}^{-\sigma} \tilde{g}_{\mu \nu}, \Phi_{i}\right)\right], \\
U(\sigma) \equiv & \mathrm{e}^{-\sigma} A(\sigma)-\mathrm{e}^{-2 \sigma} F(A(\sigma)) .
\end{aligned}
$$

Let us now consider the gravitational wave, based on the action (52) in the Einstein frame by considering the perturbation of the background metric, $\tilde{g}_{\mu \nu}=\tilde{g}_{\mu \nu}^{(0)}$ as $\tilde{g}_{\mu \nu}=$ $\tilde{g}_{\mu \nu}^{(0)}+\tilde{h}_{\mu \nu}$ in the Einstein equation, so we have

$$
\begin{aligned}
\tilde{R}_{\mu \nu}-\frac{1}{2} \tilde{g}_{\mu \nu} \tilde{R}= & 3 \partial_{\mu} \sigma \partial_{\nu} \sigma+\tilde{g}_{\mu \nu}\left(-\frac{3}{2} \partial_{\rho} \sigma \partial^{\rho} \sigma-U(\sigma)\right) \\
& +\kappa^{2} \tilde{T}_{\text {matter } \mu \nu} .
\end{aligned}
$$

Here, the matter energy-momentum tensor $\tilde{T}_{\text {matter }}^{\mu \nu}$ in the Einstein frame is

$$
\tilde{T}_{\text {matter }}^{\mu \nu} \equiv \frac{2}{\sqrt{-\tilde{g}}} \frac{\partial\left(\sqrt{-\tilde{g}} \mathrm{e}^{-2 \sigma} \mathcal{L}_{\text {matter }}\left(\mathrm{e}^{-\sigma} \tilde{g}_{\mu \nu}, \Phi_{i}\right)\right)}{\partial \tilde{g}_{\mu \nu}} .
$$

If the matter fluids are minimally coupled with gravity, that is, if the matter Lagrangian is of the form $\mathcal{L}_{\text {matter }}\left(\mathrm{e}^{-\sigma} \tilde{g}_{\mu \nu}, \Phi_{i}\right)=\mathcal{L}_{\text {matter }}\left(g_{\mu \nu}, \Phi_{i}\right)$, so that it does not include any derivative of the metric $g_{\mu \nu}$, the matter energymomentum tensor $\tilde{T}_{\text {matter }}^{\mu \nu}$ in the Einstein frame is related with the matter energy-momentum tensor $T_{\text {matter }}^{\mu \nu}$ in the original Jordan frame as $\tilde{T}_{\text {matter }}^{\mu \nu}=\mathrm{e}^{-3 \sigma} T_{\text {matter }}^{\mu \nu}$, that is, $\tilde{T}_{\text {matter } \mu \nu}=\mathrm{e}^{-\sigma} T_{\text {matter } \mu \nu}$. When we consider the gravitational wave, we often use the transverse and traceless gauge conditions. Since we are considering the scale transformation (51), if $h_{\mu \nu}$, which is defined as the fluctuation from the background metric $g_{\mu \nu}=g_{\mu \nu}^{(0)}$ as $g_{\mu \nu}=$ $g_{\mu \nu}^{(0)}+h_{\mu \nu}$ in the original frame in the action (47), it satisfies the transverse and traceless gauge conditions,

$$
\nabla^{\mu} h_{\mu \nu}=g^{(0) \mu \nu} h_{\mu \nu}=0 .
$$

However, the scale transformed fluctuation $\tilde{h}_{\mu \nu}=\mathrm{e}^{\sigma} h_{\mu \nu}$ does not always satisfy the first condition in Eq. (55), although the second condition is trivially satisfied, $\tilde{g}^{(0) \mu \nu} \tilde{h}_{\mu \nu}=\mathrm{e}^{-\sigma} g^{(0) \mu \nu} \mathrm{e}^{\sigma} h_{\mu \nu}=g^{(0) \mu \nu} h_{\mu \nu}=0$. For the first condition in Eq. (55), under the scale transformation, we find

$$
\begin{aligned}
\tilde{\nabla}^{\mu} \tilde{h}_{\mu}{ }^{\nu}= & \mathrm{e}^{-\sigma} \nabla^{\mu} h_{\mu \nu}+4 \mathrm{e}^{-\sigma} g^{(0) \mu \tau} g^{(0) \nu \rho} \sigma_{, \tau} h_{\mu \rho} \\
& -\mathrm{e}^{-\sigma} g^{(0) \nu \rho} \sigma_{, \rho} g^{(0) \mu \tau} h_{\mu \tau}=4 \mathrm{e}^{-\sigma} g^{(0) \mu \tau} g^{(0) \nu \rho} \sigma_{, \tau} h_{\mu \rho} .
\end{aligned}
$$

Then, if we assume that we have a homogeneous and isometric background metric, $\sigma$ depends solely on the cosmological time $t$, and also $g_{t i}^{(0)}=0$. In effect, if we consider the perturbation with $h_{t \mu}=0$ since we are considering the massless spin-two mode, we find

$$
\tilde{\nabla}^{\mu} \tilde{h}_{\mu}{ }^{\nu}=\tilde{g}^{(0) \mu \nu} \tilde{h}_{\mu \nu}=0 .
$$

Therefore, the gauge conditions in (55) for the graviton are not changed by the scale transformation under consideration,

$$
\tilde{\nabla}^{\mu} \tilde{h}_{\mu \nu}=\tilde{g}^{(0) \mu \nu} \tilde{h}_{\mu \nu}=0 .
$$

Then, under condition (58), the equation for the gravitational wave can be written as follows:

$$
\begin{aligned}
0= & \frac{1}{2 \kappa^{2}}\left(-\frac{1}{2}\left(-\tilde{\square}^{(0)} \tilde{h}_{\mu \nu}-2 \tilde{R}_{\nu}^{(0) \lambda}{ }_{\nu}{ }_{\mu} \tilde{h}_{\lambda \rho}+\tilde{R}^{(0) \rho}{ }_{\mu} \tilde{h}_{\rho \nu}\right.\right. \\
& \left.\left.+\tilde{R}_{\nu}^{(0) \rho} \tilde{h}_{\rho \mu}\right)+\frac{1}{2} R^{(0)} h_{\mu \nu}-\frac{1}{2} \tilde{g}_{\mu \nu}^{(0)} \tilde{h}_{\rho \sigma} \tilde{R}^{(0) \rho \sigma}\right) \\
& +\tilde{h}_{\mu \nu}\left(-\frac{3}{2} \partial_{\rho} \sigma \partial^{\rho} \sigma-U(\sigma)\right)+\frac{3}{2} \tilde{g}_{\mu \nu}^{(0)} \partial^{\rho} \sigma \partial^{\tau} \sigma \tilde{h}_{\rho \tau} \\
& +\frac{1}{2} \frac{\partial \tilde{T}_{\operatorname{matter} \mu \nu}}{\partial \tilde{g}_{\rho \tau}} \tilde{h}_{\rho \tau} .
\end{aligned}
$$

We are now interested in the massless spin-two mode, which satisfies

$$
\begin{aligned}
\tilde{h}_{i t} & =\tilde{h}_{t i}=h_{i t}=h_{t i}=0, \\
\sum_{i=1,2,3} \tilde{h}_{i i} & =\sum_{i=1,2,3} h_{i i}=0, \quad i=1,2,3, \quad \tilde{h}_{t t}=h_{t t}=0 .
\end{aligned}
$$

In the spatially flat FRW universe in the Einstein frame,

$$
d \tilde{s}^{2} \equiv \mathrm{e}^{\sigma} d s^{2}=-d \tilde{t}^{2}+\tilde{a}(\tilde{t})^{2} \sum_{i=1,2,3}\left(d x^{i}\right)^{2},
$$

where $d \tilde{t} \equiv \mathrm{e}^{\frac{\sigma}{2}} d t$ and $\tilde{a}(\tilde{t}) \equiv \mathrm{e}^{\frac{\sigma}{2}} a(t)$; because of the isometry in the spacial part, we may assume

$$
\frac{\partial \tilde{T}_{\text {matter } t t}}{\partial \tilde{g}_{i j}} \propto \delta^{i j}, \quad \frac{\partial \tilde{T}_{\text {matter } t k}}{\partial \tilde{g}_{i j}}=\frac{\partial \tilde{T}_{\text {matter } k t}}{\partial \tilde{g}_{i j}}=0 .
$$

We may further assume that the matter energy-momentum tensor (54) in the Einstein frame has the following perfect fluid form:

$$
\tilde{T}_{\text {matter } \mu \nu}=\tilde{\rho} \tilde{U}_{\mu} \tilde{U}_{\nu}+\tilde{p} \tilde{\gamma}_{\mu \nu} .
$$

Here $\left(\tilde{U}^{\mu}\right)$ is the four velocity of the matter fluid and we now assume $\tilde{U}^{0}=1$ and $\tilde{U}^{i}=0$. In Eq. (63), $\tilde{\gamma}_{\mu \nu}$ is the projection tensor to the spatial directions perpendicular to $\tilde{U}^{\mu}$, 


$$
\tilde{\gamma}_{\mu \nu}=\tilde{g}_{\mu \nu}+\tilde{U}_{\mu} \tilde{U}_{\nu}
$$

We now also assume that the matter fluid minimally couples with the metric $\tilde{g}_{\mu \nu}$, that is, the coupling between the matter fluids and the metric does not include the derivative of the metric. Then, under the perturbation $\tilde{g}_{\mu \nu}=\tilde{g}_{\mu \nu}^{(0)}+\tilde{h}_{\mu \nu}$, we find

$$
\delta \tilde{\rho}=\tilde{\rho}^{\mu \nu} \tilde{h}_{\mu \nu}, \quad \delta \tilde{p}=\tilde{p}^{\mu \nu} \tilde{h}_{\mu \nu} .
$$

On the other hand, the variation of $\tilde{U}_{\mu}$ is given by using the condition $\tilde{U}^{\mu} \tilde{U}_{\mu}=-1$, that is,

$$
0=2\left(\delta \tilde{U}^{\mu}\right)+\tilde{U}^{\mu} \tilde{U}^{\nu} \tilde{h}_{\mu \nu}=\tilde{U}^{\mu}\left(2 \tilde{g}_{\mu \nu}^{(0)} \delta \tilde{U}^{\nu}+\tilde{h}_{\mu \nu} \tilde{U}^{\nu}\right),
$$

and hence we have

$$
\delta \tilde{U}^{\mu}=-\frac{1}{2} \tilde{g}^{(0) \mu \rho}\left(\tilde{h}_{\rho \nu} \tilde{U}^{\nu}+l_{\rho}\right) .
$$

Here $l_{\mu}$ is an arbitrary vector which satisfies the condition $\tilde{U}^{\mu} l_{\mu}=0$, but we choose $l_{\mu}=0$ by assuming the isometry. Because of the isometry, we may assume $\rho^{i j}$ and $p^{i j}$ are proportional to $\delta^{i j}$, Then the $(t, t)$ and $(t, i)$ components of the equation for the gravitational wave (59) are trivially satisfied and the $(i, j)$ component is given by

$$
\begin{aligned}
0= & \frac{1}{2 \kappa^{2}}\left(\frac{1}{2}\left(-\partial_{\tilde{t}}^{2} \tilde{h}_{i j}+\tilde{a}^{-2} \triangle \tilde{h}_{i j}\right)\right. \\
& \left.+\left(3 \frac{d \tilde{H}}{d \tilde{t}}+4 \tilde{H}^{2}\right) \tilde{h}_{i j}+\tilde{h}_{i j}\left(\frac{3}{2}\left(\frac{d \sigma}{d \tilde{t}}\right)^{2}-U(\sigma)\right)\right) .
\end{aligned}
$$

We may rewrite Eq. (68) in the original Jordan frame, and since $\tilde{h}_{\mu \nu}=\mathrm{e}^{\sigma} h_{\mu \nu}$ and $\tilde{a}=\mathrm{e}^{\frac{\sigma}{2}} a$, we find the following:

$$
\begin{aligned}
\frac{\partial}{\partial \tilde{t}}= & \mathrm{e}^{-\frac{\sigma}{2}} \frac{\partial}{\partial t}, \quad \frac{\partial^{2}}{\partial \tilde{t}^{2}}=\mathrm{e}^{-\sigma}\left(\frac{\partial^{2}}{\partial t^{2}}-\frac{1}{2} \frac{d \sigma}{d t} \frac{\partial}{\partial t}\right), \\
\tilde{H}= & \frac{1}{\tilde{a}} \frac{d \tilde{a}}{d \tilde{t}}=\mathrm{e}^{-\frac{\sigma}{2}}\left(\frac{1}{2} \frac{d \sigma}{d t}+H\right), \\
\frac{d \tilde{H}}{d \tilde{t}}= & \mathrm{e}^{-\tilde{\sigma}}\left(\frac{1}{2} \frac{d^{2} \sigma}{d t^{2}}+\frac{1}{4}\left(\frac{d \sigma}{d t}\right)^{2}+\frac{d H}{d t}\right), \\
\frac{\partial^{2} \tilde{h}_{i j}}{\partial \tilde{t}^{2}}= & \frac{\partial^{2} h_{i j}}{\partial t^{2}}+\frac{d^{2} \sigma}{d t^{2}} h_{i j}+\left(\frac{d \sigma}{d t}\right)^{2} h_{i j}+2 \frac{d \sigma}{d t} \frac{\partial h_{i j}}{\partial t} \\
& -\frac{1}{2}\left(\frac{d \sigma}{d t}\right)^{2} \frac{\partial h_{i j}}{\partial t}-\frac{1}{2} \frac{d \sigma}{d t} \frac{\partial h_{i j}}{\partial t} \\
= & \frac{\partial^{2} h_{i j}}{\partial t^{2}}+\frac{d^{2} \sigma}{d t^{2}} h_{i j}+\frac{1}{2}\left(\frac{d \sigma}{d t}\right)^{2} h_{i j}+\frac{3}{2} \frac{d \sigma}{d t} \frac{\partial h_{i j}}{\partial t} .
\end{aligned}
$$

Then, due to the fact that $\tilde{p}=\mathrm{e}^{-2 \sigma} p$ from Eq. (63) $\left(\tilde{T}_{\text {matter } \mu \nu}=\mathrm{e}^{-\sigma} T_{\text {matter } \mu \nu}\right.$ and $\left.\tilde{\gamma}_{\mu \nu}=\mathrm{e}^{\sigma} \gamma_{\mu \nu}\right)$, Eq. (68) can be rewritten as follows:

$$
\begin{aligned}
0= & \frac{1}{2 \kappa^{2}}\left(\frac{1}{2}\left(-\partial_{t}^{2} h_{i j}+\frac{3}{2} \dot{\sigma} \partial_{t} h_{i j}+\left(\ddot{\sigma}+\dot{\sigma}^{2}\right) h_{i j}+a^{-2} \triangle h_{i j}\right)\right. \\
& \left.+\left(3 \dot{H}+4 H^{2}+\frac{3}{2} \ddot{\sigma}+\frac{13}{4} \dot{\sigma}^{2}+4 \dot{\sigma} H-\mathrm{e}^{\sigma} U(\sigma)\right) h_{i j}\right) \\
& +\frac{1}{2} \mathrm{e}^{-\sigma} p h_{i j} .
\end{aligned}
$$

We should note that $\mathrm{e}^{\sigma}$ is given in Eq. (49) and $U(\sigma)$ is given in Eq. (52). Then in terms of the Jordan frame, we find

$$
\begin{aligned}
\sigma & =\ln \left(F^{\prime}(R)\right), \\
U(\sigma) & =\mathrm{e}^{-\sigma} R-\mathrm{e}^{-2 \sigma} F(R)=\frac{R F^{\prime}(R)-F(R)}{F^{\prime}(R)^{2}} .
\end{aligned}
$$

From Eq. (70), it is clear that if $\dot{\sigma}>0$, the gravitational wave is enhanced and if $\dot{\sigma}<0$, dissipation of the gravity wave occurs. The enhancement or the dissipation of the gravity wave occurs due to the term $\sim \frac{3}{2} \dot{\sigma} \partial_{t} h_{i j}$ in Eq. (70), which includes the first derivative of $h_{i j}$. The enhancement or the dissipation of the gravity wave occurs as an effect originating from the scale transformation, $\tilde{h}_{\mu \nu}=\mathrm{e}^{\sigma} h_{\mu \nu}$.

Comparing the propagation of the gravitational wave in $F(R)$ gravity with the gravitational wave in the Einstein Chern-Simons gravity, it is apparent that in the $F(R)$ gravity case, there is no difference in the propagation between the right-handed mode and the left-handed mode in helicity, an effect which occurs due to the fact that there is no violation of parity in $F(R)$ gravity. However, it is expected that the presence of the Chern-Simons term in vacuum $F(R)$ gravity may induce nontrivial phenomena. We should also note that there appears to be a dissipation or enhancement term coming from the Chern-Simons term in the Chern-Simons Einstein gravity; a similar term also appears in the $F(R)$ gravity.

\section{CONCLUSIONS}

In this work we studied the propagation of gravitational waves in the Chern-Simons axion Einstein gravity context. Our aim was to examine whether it is possible to reveal at a quantitative level, any significant difference between the two polarization modes. We performed the study assuming a flat FRW background, and by studying the tensor perturbations of the metric, we demonstrated that the + mode and $\times$ mode cannot be independent from each other but they appear only as the combination of the right-handed mode or the left-handed mode and the dispersion relations of the right-handed mode and the left-handed mode are different, even for the simplest form of the Chern-Simons scalar axionic coupling. This is because the Chern-Simons scalar term breaks parity and therefore the model is chiral. We may expect that the difference of the polarization could be detected by LIGO and forthcoming LISA Collaboration. Also, the polarization of the primordial gravity waves 
influences the polarization of cosmic microwave background, E mode, and B mode in the early Universe [60]. It is conceivable that more complex axion scalar couplings to the Chern-Simons term may further perplex the dispersion relations for the two polarization modes. For the lowfrequency mode, the Chern-Simons scalar coupling works against the dissipation in the right-handed mode, which works to increase the dissipation for the left-handed mode. On the other hand, for the high frequency mode, the ChernSimons scalar coupling makes the dissipation of the gravitational wave four times stronger than that in case of the standard Einstein gravity. $U_{0}=0$ in (46). In addition to the above findings, we demonstrated that there exists a nontrivial mixing between the two different polarization modes, which strongly suggests differences between the standard scalar axion Einstein gravity and the ChernSimons axion Einstein gravity. Just for comparison, we also investigated the propagation of the gravitational wave in the $F(R)$ gravity model and found that there is no difference between the right-handed mode and the lefthanded mode, which is, of course, because the model does not violate the parity. We should also note that the $F(R)$ gravity includes a scalar propagating mode as in the Einstein frame action (52). Even in the Chern-Simons axion Einstein gravity, as clear from the action (12), there appears a propagating scalar mode. Both of the scalar modes are massive and there is not an explicit difference. However, regarding the gravitational waves, in the $f(R)$ gravity case, one has a scalar component of the gravity waves, which is, however, not present in the Chern-Simons axion Einstein case. The only effect of the Chern-Simons term is to discriminate between the two tensor modes of the gravitational wave, and in fact this is the new feature that the Chern-Simons term induces, and it is a challenge for the gravitational wave astronomy to find any parity violating gravitational modes. A highly nontrivial task is to investigate the $F(R)$ gravity extension of the axion ChernSimons gravity. We aim to study this case in a future work.

\section{ACKNOWLEDGMENTS}

This work is supported by MINECO (Spain), FIS201676363-P, and by project 2017 SGR247 (AGAUR, Catalonia) (S. D. O). This work is also supported by MEXT KAKENHI Grant-in-Aid for Scientific Research on Innovative Areas "Cosmic Acceleration" No. $15 \mathrm{H} 05890$ (S. N.) and the JSPS Grant-in-Aid for Scientific Research (C) No. 18K03615 (S. N.). This work is supported by the DAAD program Hochschulpartnerschaften mit Griechenland (Projekt No. 57340132) (V. K. O). V. K. O is indebted to Professor K. Kokkotas for his hospitality in the IAAT, University of Tübingen. The work of A.P. is performed according to the Russian Government Program of Competitive Growth of Kazan Federal University. The work of A.P. was also supported by the Russian Foundation for Basic Research Grant No 19-02-00496.

\section{APPENDIX: DETAILED FORM OF EINSTEIN TENSOR COMPONENTS}

In this Appendix we present the explicit form of the $(t, t),(i, j)$, and $(t, i)$ components of the Einstein tensor of Eq. (22) in the FRW background (15), which are

$$
\begin{aligned}
G_{t t}=-\dot{H} h_{t t} & +H^{2}\left[-3 h_{t t}+\frac{2}{a^{2}}\left(h_{x x}+h_{y y}+h_{z z}\right)\right]+\frac{H}{a^{2}}\left[-\frac{\partial}{\partial t}\left(h_{x x}+h_{y y}+h_{z z}\right)+2\left(\frac{\partial h_{t x}}{\partial x}+\frac{\partial h_{t y}}{\partial y}+\frac{\partial h_{t z}}{\partial z}\right)\right] \\
+\frac{1}{a^{4}}[ & \left.\frac{1}{2}\left\{\frac{\partial^{2}}{\partial x^{2}}\left(h_{y y}+h_{z z}\right)+\frac{\partial^{2}}{\partial y^{2}}\left(h_{x x}+h_{z z}\right)+\frac{\partial^{2}}{\partial z^{2}}\left(h_{x x}+h_{y y}\right)\right\}-\frac{\partial^{2} h_{x y}}{\partial x \partial y}-\frac{\partial^{2} h_{x z}}{\partial x \partial z}-\frac{\partial^{2} h_{y z}}{\partial y \partial z}\right], \\
G_{x x}= & \dot{H}\left[a^{2} h_{t t}-h_{y y}-h_{z z}+\frac{8 \dot{U}}{a}\left(\frac{\partial h_{x z}}{\partial y}-\frac{\partial h_{x y}}{\partial z}\right)\right]+H^{2}\left(3 h_{t t}-h_{y y}-h_{z z}\right) \\
& +H\left[a^{2} \frac{\partial h_{t t}}{\partial t}-\frac{1}{2} \frac{\partial\left(h_{y y}+h_{z z}\right)}{\partial t}-\frac{\partial h_{t y}}{\partial y}-\frac{\partial h_{t z}}{\partial z}+\frac{8 \ddot{U}}{a}\left(\frac{\partial h_{x z}}{\partial y}-\frac{\partial h_{x y}}{\partial z}\right)+\frac{8 \dot{U}}{a}\left(\frac{\partial^{2} h_{x z}}{\partial y \partial t}-\frac{\partial^{2} h_{x y}}{\partial z \partial t}\right)\right] \\
& +\frac{1}{2}\left(\frac{\partial^{2}\left(h_{y y}+h_{z z}\right)}{\partial t^{2}}+\frac{\partial^{2} h_{t t}}{\partial y^{2}}+\frac{\partial^{2} h_{t t}}{\partial z^{2}}\right)-\frac{\partial^{2} h_{t y}}{\partial y \partial t}-\frac{\partial^{2} h_{t z}}{\partial z \partial t}+\frac{4 \ddot{U}}{a}\left(\frac{\partial^{2} h_{t z}}{\partial y \partial x}-\frac{\partial^{2} h_{t y}}{\partial z \partial x}+\frac{\partial^{2} h_{x y}}{\partial z \partial t}-\frac{\partial^{2} h_{x z}}{\partial y \partial t}\right) \\
& +\frac{4 \dot{U}}{a}\left(\frac{\partial^{3} h_{t z}}{\partial y \partial x \partial t}-\frac{\partial^{3} h_{t y}}{\partial z \partial x \partial t}-\frac{\partial^{3} h_{x z}}{\partial y \partial t^{2}}+\frac{\partial^{3} h_{x y}}{\partial z \partial t^{2}}\right)+\frac{1}{a^{2}}\left(\frac{\partial^{2} h_{y z}}{\partial z \partial y}-\frac{1}{2} \frac{\partial^{2} h_{y y}}{\partial z^{2}}-\frac{1}{2} \frac{\partial^{2} h_{z z}}{\partial y^{2}}\right) \\
& +\frac{4 \dot{U}}{a^{3}}\left(\frac{\partial^{3} h_{x z}}{\partial y^{3}}-\frac{\partial^{3} h_{x y}}{\partial z^{3}}-\frac{\partial^{3} h_{x y}}{\partial z \partial y^{2}}+\frac{\partial^{3} h_{x z}}{\partial y \partial z^{2}}-\frac{\partial^{3} h_{y z}}{\partial x \partial y^{2}}+\frac{\partial^{3} h_{y z}}{\partial x \partial z^{2}}+\frac{\partial^{3}\left(h_{y y}-h_{z z}\right)}{\partial x \partial y \partial z}\right),
\end{aligned}
$$




$$
\begin{aligned}
& G_{y y}=\dot{H}\left[a^{2} h_{t t}-h_{x x}-h_{z z}-\frac{8 \dot{U}}{a}\left(\frac{\partial h_{y z}}{\partial x}-\frac{\partial h_{x y}}{\partial z}\right)\right]+H^{2}\left(3 h_{t t}-h_{x x}-h_{z z}\right) \\
& +H\left[a^{2} \frac{\partial h_{t t}}{\partial t}-\frac{1}{2} \frac{\partial\left(h_{x x}+h_{z z}\right)}{\partial t}-\frac{\partial h_{t x}}{\partial x}-\frac{\partial h_{t z}}{\partial z}-\frac{8 \ddot{U}}{a}\left(\frac{\partial h_{y z}}{\partial x}-\frac{\partial h_{x y}}{\partial z}\right)-\frac{8 \dot{U}}{a}\left(\frac{\partial^{2} h_{y z}}{\partial x \partial t}-\frac{\partial^{2} h_{x y}}{\partial z \partial t}\right)\right] \\
& +\frac{1}{2}\left(\frac{\partial^{2}\left(h_{x x}+h_{z z}\right)}{\partial t^{2}}+\frac{\partial^{2} h_{t t}}{\partial x^{2}}+\frac{\partial^{2} h_{t t}}{\partial z^{2}}\right)-\frac{\partial^{2} h_{t x}}{\partial x \partial t}-\frac{\partial^{2} h_{t z}}{\partial z \partial t}-\frac{4 \ddot{U}}{a}\left(\frac{\partial^{2} h_{t z}}{\partial y \partial x}-\frac{\partial^{2} h_{t x}}{\partial z \partial y}+\frac{\partial^{2} h_{x y}}{\partial z \partial t}-\frac{\partial^{2} h_{y z}}{\partial x \partial t}\right) \\
& -\frac{4 \dot{U}}{a}\left(\frac{\partial^{3} h_{t z}}{\partial x \partial y \partial t}-\frac{\partial^{3} h_{t x}}{\partial z \partial y \partial t}-\frac{\partial^{3} h_{y z}}{\partial x \partial t^{2}}+\frac{\partial^{3} h_{x y}}{\partial z \partial t^{2}}\right)+\frac{1}{a^{2}}\left(\frac{\partial^{2} h_{x z}}{\partial z \partial x}-\frac{1}{2} \frac{\partial^{2} h_{x x}}{\partial z^{2}}-\frac{1}{2} \frac{\partial^{2} h_{z z}}{\partial x^{2}}\right) \\
& -\frac{4 \dot{U}}{a^{3}}\left(\frac{\partial^{3} h_{y z}}{\partial x^{3}}-\frac{\partial^{3} h_{x y}}{\partial z^{3}}-\frac{\partial^{3} h_{x y}}{\partial z \partial x^{2}}+\frac{\partial^{3} h_{y z}}{\partial x \partial z^{2}}-\frac{\partial^{3} h_{x z}}{\partial y \partial x^{2}}+\frac{\partial^{3} h_{x z}}{\partial y \partial z^{2}}+\frac{\partial^{3}\left(h_{x x}-h_{z z}\right)}{\partial x \partial y \partial z}\right), \\
& G_{z z}=\dot{H}\left[a^{2} h_{t t}-h_{x x}-h_{y y}+\frac{8 \dot{U}}{a}\left(\frac{\partial h_{y z}}{\partial x}-\frac{\partial h_{x z}}{\partial y}\right)\right]+H^{2}\left(3 h_{t t}-h_{x x}-h_{y y}\right) \\
& +H\left[a^{2} \frac{\partial h_{t t}}{\partial t}-\frac{1}{2} \frac{\partial\left(h_{x x}+h_{y y}\right)}{\partial t}-\frac{\partial h_{t x}}{\partial x}-\frac{\partial h_{t y}}{\partial y}+\frac{8 \ddot{U}}{a}\left(\frac{\partial h_{y z}}{\partial x}-\frac{\partial h_{x z}}{\partial y}\right)+\frac{8 \dot{U}}{a}\left(\frac{\partial^{2} h_{y z}}{\partial x \partial t}-\frac{\partial^{2} h_{x z}}{\partial y \partial t}\right)\right] \\
& +\frac{1}{2}\left(\frac{\partial^{2}\left(h_{x x}+h_{y y}\right)}{\partial t^{2}}+\frac{\partial^{2} h_{t t}}{\partial x^{2}}+\frac{\partial^{2} h_{t t}}{\partial y^{2}}\right)-\frac{\partial^{2} h_{t x}}{\partial x \partial t}-\frac{\partial^{2} h_{t y}}{\partial y \partial t}+\frac{4 \ddot{U}}{a}\left(\frac{\partial^{2} h_{t y}}{\partial z \partial x}-\frac{\partial^{2} h_{t x}}{\partial z \partial y}+\frac{\partial^{2} h_{x z}}{\partial y \partial t}-\frac{\partial^{2} h_{y z}}{\partial x \partial t}\right) \\
& +\frac{4 \dot{U}}{a}\left(\frac{\partial^{3} h_{t y}}{\partial x \partial z \partial t}-\frac{\partial^{3} h_{t x}}{\partial y \partial z \partial t}-\frac{\partial^{3} h_{y z}}{\partial x \partial t^{2}}+\frac{\partial^{3} h_{x z}}{\partial y \partial t^{2}}\right)+\frac{1}{a^{2}}\left(\frac{\partial^{2} h_{x y}}{\partial y \partial x}-\frac{1}{2} \frac{\partial^{2} h_{x x}}{\partial y^{2}}-\frac{1}{2} \frac{\partial^{2} h_{y y}}{\partial x^{2}}\right) \\
& +\frac{4 \dot{U}}{a^{3}}\left(\frac{\partial^{3} h_{y z}}{\partial x^{3}}-\frac{\partial^{3} h_{x z}}{\partial y^{3}}-\frac{\partial^{3} h_{x z}}{\partial y \partial x^{2}}+\frac{\partial^{3} h_{y z}}{\partial x \partial y^{2}}-\frac{\partial^{3} h_{x y}}{\partial z \partial x^{2}}+\frac{\partial^{3} h_{x y}}{\partial z \partial y^{2}}+\frac{\partial^{3}\left(h_{x x}-h_{y y}\right)}{\partial x \partial y \partial z}\right), \\
& G_{t x}=H\left[\frac{\partial h_{t t}}{\partial x}+\frac{1}{a^{2}}\left(\frac{\partial h_{x y}}{\partial y}+\frac{\partial h_{x z}}{\partial z}-\frac{\partial h_{z z}}{\partial x}-\frac{\partial h_{y y}}{\partial x}\right)\right. \\
& \left.+\frac{4 \dot{U}}{a^{3}}\left(\frac{\partial^{2} h_{y z}}{\partial y^{2}}-\frac{\partial^{2} h_{y z}}{\partial z^{2}}+\frac{\partial^{2} h_{x z}}{\partial x \partial y}-\frac{\partial^{2} h_{x y}}{\partial x \partial z}+\frac{\partial^{2} h_{z z}}{\partial y \partial z}-\frac{\partial^{2} h_{y y}}{\partial y \partial z}\right)\right] \\
& +\frac{1}{2 a^{2}}\left(\frac{\partial^{2} h_{t x}}{\partial y^{2}}+\frac{\partial^{2} h_{t x}}{\partial z^{2}}+\frac{\partial^{2}\left(h_{y y}+h_{z z}\right)}{\partial x \partial t}-\frac{\partial^{2} h_{x y}}{\partial t \partial y}-\frac{\partial^{2} h_{x z}}{\partial t \partial z}-\frac{\partial^{2} h_{t y}}{\partial x \partial y}-\frac{\partial^{2} h_{t z}}{\partial x \partial z}\right) \\
& +\frac{2 \dot{U}}{a^{3}}\left(\frac{\partial^{3} h_{t z}}{\partial y^{3}}-\frac{\partial^{3} h_{t y}}{\partial z^{3}}-\frac{\partial^{3} h_{x z}}{\partial t \partial x \partial y}+\frac{\partial^{3} h_{x y}}{\partial t \partial x \partial z}+\frac{\partial^{3}\left(h_{y y}-h_{z z}\right)}{\partial t \partial y \partial z}\right. \\
& \left.-\frac{\partial^{3} h_{y z}}{\partial t \partial y^{2}}+\frac{\partial^{3} h_{y z}}{\partial t \partial z^{2}}+\frac{\partial^{3} h_{t z}}{\partial y \partial x^{2}}-\frac{\partial^{3} h_{t y}}{\partial z \partial x^{2}}-\frac{\partial^{3} h_{t y}}{\partial z \partial y^{2}}+\frac{\partial^{3} h_{t z}}{\partial y \partial z^{2}}\right), \\
& G_{t y}=H\left[\frac{\partial h_{t t}}{\partial y}+\frac{1}{a^{2}}\left(\frac{\partial h_{x y}}{\partial x}+\frac{\partial h_{y z}}{\partial z}-\frac{\partial h_{z z}}{\partial y}-\frac{\partial h_{x x}}{\partial y}\right)\right. \\
& \left.-\frac{4 \dot{U}}{a^{3}}\left(\frac{\partial^{2} h_{x z}}{\partial x^{2}}-\frac{\partial^{2} h_{x z}}{\partial z^{2}}+\frac{\partial^{2} h_{y z}}{\partial x \partial y}-\frac{\partial^{2} h_{x y}}{\partial y \partial z}+\frac{\partial^{2} h_{z z}}{\partial x \partial z}-\frac{\partial^{2} h_{x x}}{\partial x \partial z}\right)\right] \\
& +\frac{1}{2 a^{2}}\left(\frac{\partial^{2} h_{t y}}{\partial x^{2}}+\frac{\partial^{2} h_{t y}}{\partial z^{2}}+\frac{\partial^{2}\left(h_{x x}+h_{z z}\right)}{\partial y \partial t}-\frac{\partial^{2} h_{x y}}{\partial t \partial x}-\frac{\partial^{2} h_{y z}}{\partial t \partial z}-\frac{\partial^{2} h_{t x}}{\partial x \partial y}-\frac{\partial^{2} h_{t z}}{\partial y \partial z}\right) \\
& -\frac{2 \dot{U}}{a^{3}}\left(\frac{\partial^{3} h_{t z}}{\partial x^{3}}-\frac{\partial^{3} h_{t x}}{\partial z^{3}}-\frac{\partial^{3} h_{y z}}{\partial t \partial x \partial y}+\frac{\partial^{3} h_{x y}}{\partial t \partial y \partial z}+\frac{\partial^{3}\left(h_{x x}-h_{z z}\right)}{\partial t \partial x \partial z}\right. \\
& \left.-\frac{\partial^{3} h_{x z}}{\partial t \partial x^{2}}+\frac{\partial^{3} h_{x z}}{\partial t \partial z^{2}}+\frac{\partial^{3} h_{t z}}{\partial x \partial y^{2}}-\frac{\partial^{3} h_{t x}}{\partial z \partial y^{2}}-\frac{\partial^{3} h_{t x}}{\partial z \partial x^{2}}+\frac{\partial^{3} h_{t z}}{\partial x \partial z^{2}}\right),
\end{aligned}
$$




$$
\begin{aligned}
& G_{t z}=H\left[\frac{\partial h_{t t}}{\partial z}+\frac{1}{a^{2}}\left(\frac{\partial h_{z y}}{\partial y}+\frac{\partial h_{x z}}{\partial x}-\frac{\partial h_{x x}}{\partial z}-\frac{\partial h_{y y}}{\partial z}\right)\right. \\
& \left.-\frac{4 \dot{U}}{a^{3}}\left(\frac{\partial^{2} h_{y x}}{\partial y^{2}}-\frac{\partial^{2} h_{y x}}{\partial x^{2}}+\frac{\partial^{2} h_{x z}}{\partial z \partial y}-\frac{\partial^{2} h_{z y}}{\partial x \partial z}+\frac{\partial^{2} h_{x x}}{\partial y \partial x}-\frac{\partial^{2} h_{y y}}{\partial y \partial x}\right)\right] \\
& +\frac{1}{2 a^{2}}\left(\frac{\partial^{2} h_{t z}}{\partial y^{2}}+\frac{\partial^{2} h_{t z}}{\partial x^{2}}+\frac{\partial^{2}\left(h_{y y}+h_{x x}\right)}{\partial z \partial t}-\frac{\partial^{2} h_{z y}}{\partial t \partial y}-\frac{\partial^{2} h_{x z}}{\partial t \partial x}-\frac{\partial^{2} h_{t y}}{\partial z \partial y}-\frac{\partial^{2} h_{t x}}{\partial x \partial z}\right) \\
& -\frac{2 \dot{U}}{a^{3}}\left(\frac{\partial^{3} h_{t x}}{\partial y^{3}}-\frac{\partial^{3} h_{t y}}{\partial x^{3}}-\frac{\partial^{3} h_{x z}}{\partial t \partial z \partial y}+\frac{\partial^{3} h_{z y}}{\partial t \partial x \partial z}+\frac{\partial^{3}\left(h_{y y}-h_{x x}\right)}{\partial t \partial y \partial x}\right. \\
& \left.-\frac{\partial^{3} h_{y x}}{\partial t \partial y^{2}}+\frac{\partial^{3} h_{y x}}{\partial t \partial x^{2}}+\frac{\partial^{3} h_{t x}}{\partial y \partial z^{2}}-\frac{\partial^{3} h_{t y}}{\partial x \partial z^{2}}-\frac{\partial^{3} h_{t y}}{\partial x \partial y^{2}}+\frac{\partial^{3} h_{t x}}{\partial y \partial x^{2}}\right), \\
& G_{x y}=\dot{H}\left[h_{x y}+\frac{4 \dot{U}}{a}\left(\frac{\partial\left(h_{x x}-h_{y y}\right)}{\partial z}+\frac{\partial h_{y z}}{\partial y}-\frac{\partial h_{x z}}{\partial x}\right)\right]+H^{2} h_{x y} \\
& +H\left[\frac{1}{2}\left(\frac{\partial h_{t x}}{\partial y}+\frac{\partial h_{t y}}{\partial x}+\frac{\partial h_{x y}}{\partial t}\right)+\frac{4 \ddot{U}}{a}\left(\frac{\partial\left(h_{x x}-h_{y y}\right)}{\partial z}+\frac{\partial h_{y z}}{\partial y}-\frac{\partial h_{x z}}{\partial x}\right)\right. \\
& \left.+\frac{4 \dot{U}}{a}\left(\frac{\partial^{2}\left(h_{x x}-h_{y y}\right)}{\partial z \partial t}+\frac{\partial^{2} h_{y z}}{\partial y \partial t}-\frac{\partial^{2} h_{x z}}{\partial x \partial t}\right)\right]+\frac{1}{2}\left(\frac{\partial^{2} h_{t x}}{\partial y \partial t}+\frac{\partial^{2} h_{t y}}{\partial x \partial t}-\frac{\partial^{2} h_{t t}}{\partial y \partial x}-\frac{\partial^{2} h_{x y}}{\partial t^{2}}\right) \\
& +\frac{2 \ddot{U}}{a}\left(\frac{\partial^{2}\left(h_{y y}-h_{x x}\right)}{\partial z \partial t}-\frac{\partial^{2} h_{t z}}{\partial x^{2}}+\frac{\partial^{2} h_{t z}}{\partial y^{2}}+\frac{\partial^{2} h_{t x}}{\partial z \partial x}-\frac{\partial^{2} h_{t y}}{\partial z \partial y}+\frac{\partial^{2} h_{x z}}{\partial x \partial t}-\frac{\partial^{2} h_{y z}}{\partial y \partial t}\right) \\
& +\frac{2 \dot{U}}{a}\left(\frac{\partial^{3}\left(h_{y y}-h_{x x}\right)}{\partial z \partial t^{2}}+\frac{\partial^{3} h_{t x}}{\partial z \partial x \partial t}-\frac{\partial^{3} h_{t y}}{\partial z \partial y \partial t}-\frac{\partial^{3} h_{t z}}{\partial x^{2} \partial t}+\frac{\partial^{3} h_{t z}}{\partial y^{2} \partial t}+\frac{\partial^{3} h_{x z}}{\partial x \partial t^{2}}-\frac{\partial^{3} h_{y z}}{\partial y \partial t^{2}}\right) \\
& +\frac{1}{2 a^{2}}\left(\frac{\partial^{2} h_{x y}}{\partial z^{2}}+\frac{\partial^{2} h_{z z}}{\partial y \partial x}-\frac{\partial^{2} h_{x z}}{\partial z \partial y}-\frac{\partial^{2} h_{y z}}{\partial z \partial x}\right)+\frac{2 \dot{U}}{a^{3}}\left(\frac{\partial^{3}\left(h_{x x}-h_{y y}\right)}{\partial z^{3}}+\frac{\partial^{3}\left(h_{z z}-h_{y y}\right)}{\partial z \partial x^{2}}\right. \\
& \left.+\frac{\partial^{3}\left(h_{x x}-h_{z z}\right)}{\partial z \partial y^{2}}+2 \frac{\partial^{3} h_{y z}}{\partial y \partial x^{2}}-2 \frac{\partial^{3} h_{x z}}{\partial x \partial y^{2}}+2 \frac{\partial^{3} h_{y z}}{\partial y \partial z^{2}}-2 \frac{\partial^{3} h_{x z}}{\partial x \partial z^{2}}\right), \\
& G_{x z}=\dot{H}\left[h_{x z}-\frac{4 \dot{U}}{a}\left(\frac{\partial\left(h_{x x}-h_{z z}\right)}{\partial y}+\frac{\partial h_{y z}}{\partial z}-\frac{\partial h_{x y}}{\partial x}\right)\right]+H^{2} h_{x z} \\
& +H\left[\frac{1}{2}\left(\frac{\partial h_{t x}}{\partial z}+\frac{\partial h_{t z}}{\partial x}+\frac{\partial h_{x z}}{\partial t}\right)-\frac{4 \ddot{U}}{a}\left(\frac{\partial\left(h_{x x}-h_{z z}\right)}{\partial y}+\frac{\partial h_{y z}}{\partial z}-\frac{\partial h_{x y}}{\partial x}\right)\right. \\
& \left.-\frac{4 \dot{U}}{a}\left(\frac{\partial^{2}\left(h_{x x}-h_{z z}\right)}{\partial y \partial t}+\frac{\partial^{2} h_{y z}}{\partial z \partial t}-\frac{\partial^{2} h_{x y}}{\partial x \partial t}\right)\right]+\frac{1}{2}\left(\frac{\partial^{2} h_{t x}}{\partial z \partial t}+\frac{\partial^{2} h_{t z}}{\partial x \partial t}-\frac{\partial^{2} h_{t t}}{\partial z \partial x}-\frac{\partial^{2} h_{x z}}{\partial t^{2}}\right) \\
& -\frac{2 \ddot{U}}{a}\left(\frac{\partial^{2}\left(h_{z z}-h_{x x}\right)}{\partial y \partial t}-\frac{\partial^{2} h_{t y}}{\partial x^{2}}+\frac{\partial^{2} h_{t y}}{\partial z^{2}}+\frac{\partial^{2} h_{t x}}{\partial y \partial x}-\frac{\partial^{2} h_{t z}}{\partial z \partial y}+\frac{\partial^{2} h_{x y}}{\partial x \partial t}-\frac{\partial^{2} h_{y z}}{\partial z \partial t}\right) \\
& -\frac{2 \dot{U}}{a}\left(\frac{\partial^{3}\left(h_{z z}-h_{x x}\right)}{\partial y \partial t^{2}}+\frac{\partial^{3} h_{t x}}{\partial y \partial x \partial t}-\frac{\partial^{3} h_{t z}}{\partial z \partial y \partial t}-\frac{\partial^{3} h_{t y}}{\partial x^{2} \partial t}+\frac{\partial^{3} h_{t y}}{\partial z^{2} \partial t}+\frac{\partial^{3} h_{x y}}{\partial x \partial t^{2}}-\frac{\partial^{3} h_{y z}}{\partial z \partial t^{2}}\right) \\
& +\frac{1}{2 a^{2}}\left(\frac{\partial^{2} h_{x z}}{\partial y^{2}}+\frac{\partial^{2} h_{y y}}{\partial z \partial x}-\frac{\partial^{2} h_{x y}}{\partial z \partial y}-\frac{\partial^{2} h_{y z}}{\partial y \partial x}\right)-\frac{2 \dot{U}}{a^{3}}\left(\frac{\partial^{3}\left(h_{x x}-h_{z z}\right)}{\partial y^{3}}+\frac{\partial^{3}\left(h_{y y}-h_{z z}\right)}{\partial y \partial x^{2}}\right. \\
& \left.+\frac{\partial^{3}\left(h_{x x}-h_{y y}\right)}{\partial y \partial z^{2}}+2 \frac{\partial^{3} h_{y z}}{\partial z \partial x^{2}}-2 \frac{\partial^{3} h_{x y}}{\partial x \partial z^{2}}+2 \frac{\partial^{3} h_{y z}}{\partial z \partial y^{2}}-2 \frac{\partial^{3} h_{x y}}{\partial x \partial y^{2}}\right),
\end{aligned}
$$




$$
\begin{aligned}
G_{y z}= & \dot{H}\left[h_{y z}+\frac{4 \dot{U}}{a}\left(\frac{\partial\left(h_{y y}-h_{z z}\right)}{\partial x}+\frac{\partial h_{x z}}{\partial z}-\frac{\partial h_{x y}}{\partial y}\right)\right]+H^{2} h_{y z} \\
& +H\left[\frac{1}{2}\left(\frac{\partial h_{t y}}{\partial z}+\frac{\partial h_{t z}}{\partial y}+\frac{\partial h_{y z}}{\partial t}\right)+\frac{4 \ddot{U}}{a}\left(\frac{\partial\left(h_{y y}-h_{z z}\right)}{\partial x}+\frac{\partial h_{x z}}{\partial z}-\frac{\partial h_{x y}}{\partial y}\right)\right. \\
& \left.+\frac{4 \dot{U}}{a}\left(\frac{\partial^{2}\left(h_{y y}-h_{z z}\right)}{\partial x \partial t}+\frac{\partial^{2} h_{x z}}{\partial z \partial t}-\frac{\partial^{2} h_{x y}}{\partial y \partial t}\right)\right]+\frac{1}{2}\left(\frac{\partial^{2} h_{t y}}{\partial z \partial t}+\frac{\partial^{2} h_{t z}}{\partial y \partial t}-\frac{\partial^{2} h_{t t}}{\partial z \partial y}-\frac{\partial^{2} h_{y z}}{\partial t^{2}}\right) \\
& +\frac{2 \ddot{U}}{a}\left(\frac{\partial^{2}\left(h_{z z}-h_{y y}\right)}{\partial x \partial t}-\frac{\partial^{2} h_{t x}}{\partial y^{2}}+\frac{\partial^{2} h_{t x}}{\partial z^{2}}+\frac{\partial^{2} h_{t y}}{\partial y \partial x}-\frac{\partial^{2} h_{t z}}{\partial z \partial x}+\frac{\partial^{2} h_{x y}}{\partial y \partial t}-\frac{\partial^{2} h_{x z}}{\partial z \partial t}\right) \\
& +\frac{2 \dot{U}}{a}\left(\frac{\partial^{3}\left(h_{z z}-h_{y y}\right)}{\partial x \partial t^{2}}+\frac{\partial^{3} h_{t y}}{\partial y \partial x \partial t}-\frac{\partial^{3} h_{t z}}{\partial z \partial x \partial t}-\frac{\partial^{3} h_{t x}}{\partial y^{2} \partial t}+\frac{\partial^{3} h_{t x}}{\partial z^{2} \partial t}+\frac{\partial^{3} h_{x y}}{\partial y \partial t^{2}}-\frac{\partial^{3} h_{x z}}{\partial z \partial t^{2}}\right) \\
& +\frac{1}{2 a^{2}}\left(\frac{\partial^{2} h_{y z}}{\partial x^{2}}+\frac{\partial^{2} h_{x x}}{\partial z \partial y}-\frac{\partial^{2} h_{x y}}{\partial z \partial x}-\frac{\partial^{2} h_{x z}}{\partial y \partial x}\right)+\frac{2 \dot{U}}{a^{3}}\left(\frac{\partial^{3}\left(h_{y y}-h_{z z}\right)}{\partial x^{3}}+\frac{\partial^{3}\left(h_{x x}-h_{z z}\right)}{\partial x \partial y^{2}}\right. \\
& \left.+\frac{\partial^{3}\left(h_{y y}-h_{x x}\right)}{\partial x \partial z^{2}}+2 \frac{\partial^{3} h_{x z}}{\partial z \partial y^{2}}-2 \frac{\partial^{3} h_{x y}}{\partial y \partial z^{2}}+2 \frac{\partial^{3} h_{x z}}{\partial z \partial x^{2}}-2 \frac{\partial^{3} h_{x y}}{\partial y \partial x^{2}}\right) .
\end{aligned}
$$

[1] D. J. E. Marsh, Phys. Rep. 643, 1 (2016).

[2] M. C. D. Marsh, H. R. Russell, A. C. Fabian, B. P. McNamara, P. Nulsen, and C. S. Reynolds, J. Cosmol. Astropart. Phys. 12 (2017) 036.

[3] S. D. Odintsov and V. K. Oikonomou, Phys. Rev. D 99, 064049 (2019).

[4] S. Nojiri, S. D. Odintsov, and V. K. Oikonomou, arXiv:1907 .01625 .

[5] S. D. Odintsov and V. K. Oikonomou, Phys. Rev. D 99, 104070 (2019).

[6] M. Cicoli, V. Guidetti, and F. G. Pedro, J. Cosmol. Astropart. Phys. 05 (2019) 046.

[7] H. Fukunaga, N. Kitajima, and Y. Urakawa, J. Cosmol. Astropart. Phys. 06 (2019) 055.

[8] A. Caputo, Phys. Lett. B 797, 134824 (2019).

[9] A. S. Sakharov and M. Y. Khlopov, Yad. Fiz. 57, 514 (1994) [Phys. At. Nucl. 57, 485 (1994)].

[10] A. S. Sakharov, D. D. Sokoloff, and M. Y. Khlopov, Yad. Fiz. 59, 1050 (1996) [Phys. At. Nucl. 59, 1005 (1996)].

[11] M. Y. Khlopov, A. S. Sakharov, and D. D. Sokoloff, Nucl. Phys. B, Proc. Suppl. 72, 105 (1999).

[12] N. Du et al. (ADMX Collaboration), Phys. Rev. Lett. 120, 151301 (2018).

[13] R. Henning et al. (ABRACADABRA Collaboration), https:// dx.doi.org/10.3204/DESY-PROC-2017-02/henning_reyco.

[14] J. L. Ouellet et al., Phys. Rev. Lett. 122, 121802 (2019).

[15] B. R. Safdi, Z. Sun, and A. Y. Chen, Phys. Rev. D 99, 123021 (2019).

[16] M. Rozner, E. Grishin, Y. B. Ginat, A. P. Igoshev, and V. Desjacques, arXiv:1904.01958.

[17] F. T. Avignone, R. J. Creswick, and J. D. Vergados, arXiv: 1801.02072 .
[18] A. Caputo, M. Regis, M. Taoso, and S. J. Witte, J. Cosmol. Astropart. Phys. 03 (2019) 027.

[19] A. Caputo, C. P. Garay, and S. J. Witte, Phys. Rev. D 98, 083024 (2018).

[20] A. B. Balakin and W. T. Ni, Classical Quantum Gravity 27, 055003 (2010).

[21] A. B. Balakin, V. V. Bochkarev, and N. O. Tarasova, Eur. Phys. J. C 72, 1895 (2012).

[22] A. B. Balakin, R. K. Muharlyamov, and A. E. Zayats, Eur. Phys. J. D 68, 159 (2014).

[23] M. Lawson, A. J. Millar, M. Pancaldi, E. Vitagliano, and F. Wilczek, arXiv:1904.11872.

[24] V. K. Oikonomou, J. D. Vergados, and C. C. Moustakidis, Nucl. Phys. B773, 19 (2007).

[25] A. Nishizawa and T. Kobayashi, Phys. Rev. D 98, 124018 (2018).

[26] P. Wagle, N. Yunes, D. Garfinkle, and L. Bieri, Classical Quantum Gravity 36, 115004 (2019).

[27] K. Yagi, N. Yunes, and T. Tanaka, Phys. Rev. Lett. 109, 251105 (2012); 116, 169902(E) (2016).

[28] K. Yagi, N. Yunes, and T. Tanaka, Phys. Rev. D 86, 044037 (2012); 89, 049902(E) (2014).

[29] C. Molina, P. Pani, V. Cardoso, and L. Gualtieri, Phys. Rev. D 81, 124021 (2010).

[30] F. Izaurieta, E. Rodriguez, P. Minning, P. Salgado, and A. Perez, Phys. Lett. B 678, 213 (2009).

[31] C. F. Sopuerta and N. Yunes, Phys. Rev. D 80, 064006 (2009).

[32] K. Konno, T. Matsuyama, and S. Tanda, Prog. Theor. Phys. 122, 561 (2009).

[33] T. L. Smith, A. L. Erickcek, R. R. Caldwell, and M. Kamionkowski, Phys. Rev. D 77, 024015 (2008). 
[34] H. J. Matschull, Classical Quantum Gravity 16, 2599 (1999).

[35] Z. Haghani, T. Harko, and S. Shahidi, Eur. Phys. J. C 77, 514 (2017).

[36] M. Satoh, S. Kanno, and J. Soda, Phys. Rev. D 77, 023526 (2008).

[37] M. Satoh and J. Soda, J. Cosmol. Astropart. Phys. 09 (2008) 019.

[38] D. Yoshida and J. Soda, Int. J. Mod. Phys. D 27, 1850096 (2018).

[39] S. Alexander, L. S. Finn, and N. Yunes, Phys. Rev. D 78, 066005 (2008).

[40] N. Yunes, R. O'Shaughnessy, B. J. Owen, and S. Alexander, Phys. Rev. D 82, 064017 (2010).

[41] S. H. Alexander and N. Yunes, Phys. Rev. D 97, 064033 (2018).

[42] J. c. Hwang and H. Noh, Phys. Rev. D 71, 063536 (2005).

[43] K. Choi, J. c. Hwang, and K. W. Hwang, Phys. Rev. D 61, 084026 (2000).

[44] K. Inomata and M. Kamionkowski, Phys. Rev. Lett. 123, 031305 (2019).

[45] M. Kamionkowski and A. Kosowsky, Phys. Rev. D 57, 685 (1998).

[46] J. R. Pritchard and M. Kamionkowski, Ann. Phys. (Amsterdam) 318, 2 (2005).
[47] D. H. Lyth, C. Quimbay, and Y. Rodriguez, J. High Energy Phys. 03 (2005) 016.

[48] S. H. S. Alexander, M. E. Peskin, and M. M. Sheikh-Jabbari, eConf C0605151, 0022 (2006).

[49] S. H. S. Alexander, M. E. Peskin, and M. M. Sheikh-Jabbari, Phys. Rev. Lett. 96, 081301 (2006).

[50] Y. Cai, Y. T. Wang, and Y. S. Piao, J. High Energy Phys. 03 (2017) 024.

[51] F. Moretti, F. Bombacigno, and G. Montani, arXiv:1906 .01899 .

[52] S. Nojiri, S. D. Odintsov, and V. K. Oikonomou, Phys. Rep. 692, 1 (2017).

[53] S. Nojiri and S. D. Odintsov, Phys. Rep. 505, 59 (2011).

[54] S. Nojiri and S. D. Odintsov, eConf C0602061, 06 (2006); Int. J. Geom. Methods Mod. Phys. 04, 115 (2007).

[55] S. Capozziello and M. De Laurentis, Phys. Rep. 509, 167 (2011).

[56] V. Faraoni and S. Capozziello, Fundamental Theories of Physics (Springer, Heidelberg, 2010).

[57] A. de la Cruz-Dombriz and D. Saez-Gomez, Entropy 14, 1717 (2012).

[58] G. J. Olmo, Int. J. Mod. Phys. D 20, 413 (2011).

[59] N. Seto and A. Taruya, Phys. Rev. D 77, 103001 (2008).

[60] J. Bielefeld and R. R. Caldwell, Phys. Rev. D 91, 123501 (2015). 